\title{
Ein Instrument zur strategischen Kompetenzentwicklung in mittelständischen gewerblichen Betrieben: Die Kompetenzmanagementtabelle (KMT)
}

\author{
Julian Decius ${ }^{1}$ (D) $\cdot$ Niclas Schaper $^{1}$ (iD \\ Angenommen: 26. März 2021 / Online publiziert: 20. April 2021 \\ (c) Der/die Autor(en) 2021
}

\section{Zusammenfassung}

Dieser Beitrag in der Zeitschrift Gruppe. Interaktion. Organisation. Zeitschrift für Angewandte Organisationspsychologie (GIO) illustriert die Implementierung und Anwendung eines Tools für die strategische Kompetenzentwicklung in mittelständischen Industriebetrieben - der Kompetenzmanagementtabelle (KMT) - anhand eines betrieblichen Fallbeispiels. Der Hintergrund ist, dass auch kleinere und mittlere produzierende Unternehmen die Personalentwicklung ihrer Beschäftigten unterstützen müssen, um langfristig in einer sich wandelnden Arbeitswelt erfolgreich zu sein. Im Rahmen einer strategischen Kompetenzentwicklung sollten dabei sowohl die Unternehmensziele als auch die aktuellen und zukünftigen Tätigkeitsanforderungen sowie der Ist-Stand an vorhandenen Kompetenzen im Betrieb in den Blick genommen werden. Wegen geringer finanzieller und personeller Ressourcen in kleinen und mittleren Unternehmen ist es sinnvoll, den Personalverantwortlichen in den Betrieben mit der KMT ein Hilfsmittel zur strategischen Kompetenzentwicklung an die Hand zu geben. Der Implementierungsbedarf und mögliche Nutzungsfelder der KMT werden anhand des Anwendungsfalls eines Betriebs mit ca. 240 Beschäftigten aus der Metallbranche dargestellt. Die KMT wurde aufbauend auf einem theoretisch fundierten Konzept entwickelt und orientiert sich direkt an den praktischen Anforderungen, die mittelständische Unternehmen an Kompetenzmanagementinstrumente stellen. Berücksichtigt werden in der KMT fachliche, interpersonelle, methodische und kenntnisbezogene Kompetenzen; zudem existieren Zusatzanalysetools für sprachliche und außerberufliche Kompetenzen. Dabei erfüllt die KMT die Kriterien der einfachen Handhabbarkeit, der zielgruppenorientierten Verständlichkeit sowie der Umsetzbarkeit als datenbankgestütztes System bzw. der Anschlussfähigkeit an bestehende Systeme. Die KMT stellt damit für Personalentwicklungs- und Führungskräfte in kleinen und mittleren Unternehmen eine wesentliche Erleichterung für das strategische Kompetenzmanagement dar.

Schlüsselwörter Strategisches Kompetenzmanagement $\cdot$ Personalentwicklung $\cdot$ Kleine und mittlere Unternehmen $(\mathrm{KMU}) \cdot$ Ist-Soll-Abgleich

Dr. Julian Decius

julian.decius@uni-paderborn.de

Prof. Dr. Niclas Schaper

niclas.schaper@uni-paderborn.de
1 Institut für Psychologie, Universität Paderborn, Warburger Straße 100, 33098 Paderborn, Deutschland 


\title{
An instrument for strategic competence development in small and medium-sized industrial enterprises: the Competency Management Tool (CMT)
}

\begin{abstract}
This article in the journal Gruppe. Interaktion. Organisation. (GIO) illustrates the implementation and application of a tool for strategic competence development in medium-sized industrial enterprises-the Competence Management Tool (CMT) - based on an enterprise case study. Even small and medium-sized industrial enterprises need to support the personnel development of their employees to be successful in the long term in a changing working world. In the context of strategic competence development, both the enterprise goals and the current and future job requirements should be taken into account, as well as the current status of employees' existing competencies. Due to low financial and human resources in small and medium-sized enterprises, it is important to provide those responsible for human resources in the enterprises with a tool for strategic competence development. The need for implementation and possible fields of application of the CMT are presented based on the use case of a company with approx. 240 employees from the metal industry. The CMT was developed on the basis of a theoretically sound concept and is oriented towards the practical requirements of medium-sized enterprises. The CMT comprises professional, interpersonal, methodological and knowledge-related competencies; there are also additional analysis tools for linguistic and off-the-job competencies. Furthermore, the CMT fulfills the criteria of simple manageability, target group-oriented comprehensibility, and implementability as a database-supported system or connectability to existing systems. The CMT thus represents a significant facilitation in the context of strategic competence management for personnel development in small and medium-sized enterprises.
\end{abstract}

Keywords Strategic Competence Management $\cdot$ Personnel Development $\cdot$ Small and medium-sized enterprises (SMEs)

\section{Herausforderungen der strategischen Kompetenzentwicklung in gewerblichen Betrieben}

Die gewerbliche Arbeitswelt ist einem stetigen Wandel unterworfen - die Unternehmen und ihre Beschäftigten sehen sich neuen Herausforderungen durch Effekte der Digitalisierung, Globalisierung und Dynamisierung der Arbeit gegenübergestellt (Cascio und Montealegre 2016; Harteis 2018; Regan und Delaney 2011; Schaper 2019b). Zum Erhalt der Wettbewerbsfähigkeit sind die Betriebe darauf angewiesen, dass sich die Beschäftigten stets weiterbilden und ihre Kompetenzen erweitern (Buller und McEvoy 2012; Kraiger und Ford 2021; Noe et al. 2014). Nachdem dieser Trend zum lebenslangen Lernen zuvor insbesondere bei größeren Unternehmen zu beobachten war, hat er in den letzten Jahren auch verstärkt bei den kleinen und mittleren Unternehmen (KMU) Einzug gehalten (Coetzer et al. 2020; Hof 2009). KMU haben eine hohe wirtschaftliche und soziale Bedeutung und tragen wesentlich zur Innovationsfähigkeit der Wirtschaft bei (Perkins 2018). Obwohl nur etwa $10 \%$ der KMU regelmäßig finanzielle Mittel in Forschung und Entwicklung investieren, präsentieren $35 \%$ der KMU kontinuierlich Produkt- oder Prozessinnovationen (Rammer et al. 2018).

Allerdings können KMU wegen geringerer Ressourcen weniger Zeit und Geld in die Personalentwicklung ihrer Mitarbeiterinnen und Mitarbeiter investieren als größere Unternehmen (Abel et al. 2016; Abel und Wagner 2017; Coetzer et al. 2017; Decius und Schaper 2017). In den Per- sonalabteilungen kleinerer Betriebe sind häufig Generalisten damit beauftragt, sich um alle anfallenden Personalangelegenheiten zu kümmern - für Personalentwicklungsspezialisten fehlt ein entsprechendes Budget. Zudem werden langfristig ausgerichtete Aufgabenbereiche oftmals von den Anforderungen des Tagesgeschäfts überlagert (Virgillito 2018). Insbesondere die Weiterbildung von an- und ungelernten Beschäftigten in KMU leidet unter diesen Restriktionen formaler Weiterbildung; sie lernen daher - teilweise aus der Not heraus - in erster Linie informell etwas dazu (Decius 2020; Decius et al. 2019). Bei dieser für den gewerblichen Bereich unverzichtbaren Beschäftigtengruppe werden Kompetenzentwicklungsausgaben oftmals mehr als monetäre Belastung denn als Investition in die zukünftig bessere Aufgabenbewältigung durch die Beschäftigten angesehen (Mesaros et al. 2009). Darüber hinaus sind extern angebotene Weiterbildungsmaßnahmen nur selten auf die Bedürfnisse der KMU zugeschnitten (Bishop 2017; Lundkvist und Gustavsson 2018; Wong und Aspinwall 2004). Langfristig wirkt sich ein solcher Mangel an Weiterbildung negativ auf die Innovationsfähigkeit von KMU aus (Hadjimanolis 1999). Zur Unterstützung der Personalentwicklung in KMU ist daher ein an diese Zielgruppe angepasstes strategisches Kompetenzmanagement notwendig, welches kostengünstig, mit geringem Aufwand umsetzbar und auch von Beschäftigten ohne Personalmanagement-Hintergrund umsetzbar ist (Moll und Weidner 2018).

Im vorliegenden Beitrag werden daher zunächst auf Basis der Literatur Kriterien zur Bewertung von Kompetenzmanagement-Tools für produzierende KMU entwickelt. 
Anschließend erfolgt ein Überblick des Forschung- und Entwicklungsstandes von Kompetenzmessinstrumenten mit Fokus auf die Zielgruppe der produzierenden KMU. Hier wird ein Bedarf zur Entwicklung eines adäquaten Kompetenzmanagement-Tools für die genannten Betriebe ersichtlich. Im Anschluss daran wird daher die Entwicklung und Implementierung der sog. Kompetenzmanagementtabelle am Fallbeispiel eines mittelständischen Betriebs aus der Metallbranche erläutert.

\section{Kriterien des strategischen Kompetenzmanagements in KMU}

Als Grundlage dieses Beitrags wird Kompetenz verstanden als ,die bei Individuen verfügbaren oder durch sie erlernbaren kognitiven Fähigkeiten und Fertigkeiten, um bestimmte Probleme zu lösen, sowie die damit verbundenen motivationalen, volitionalen und sozialen Bereitschaften und Fähigkeiten, um die Problemlösungen in variablen Situationen erfolgreich und verantwortungsvoll nutzen zu können“ (Weinert 2001, S. 27-28). Dies ist wichtig zu betonen, da diverse Perspektiven auf den Kompetenzbegriff existieren (siehe Stevens 2013, für einen Überblick). Einige Forschende nehmen sogar an, dass der Terminus „Kompetenz“ keine (übergeordnete) Bedeutung habe außerhalb der spezifischen Definition, die man gerade verwendet (Shippmann et al. 2000, S. 706) - es herrscht somit alles andere als ein Konsens darüber, was Kompetenz ist.

Gemäß Horvat et al. (2018) enthält das Konzept des strategischen Kompetenzmanagements drei wesentliche Bestandteile: Die Formulierung spezifischer Kompetenzanforderungen, die Erfassung und Dokumentation der bestehenden Kompetenzen im Unternehmen, sowie der Abgleich der vorhandenen Kompetenzen mit den künftigen Kompetenzanforderungen. Dementsprechend solle das Kompetenzmanagement eine strategische Ausrichtung besitzen, sowie proaktiv, vorausschauend und als kontinuierlicher/ iterativer Prozess umgesetzt werden. Ziel ist eine Verzahnung der ,strategische[n] Gestaltungsebene der organisatorischen Kompetenz [mit der] Gestaltungsebene der individuellen Kompetenzen von Beschäftigten“ (Horvat et al. 2018, S. 33).

Eine Analyse der bisherigen praxisnahen Forschungsliteratur zum arbeitsbezogenen Lernen, zur betrieblichen Weiterbildung und zum strategischen Kompetenzmanagement in der Zielgruppe der mittelständischen Betriebe des verarbeitenden Gewerbes ergab folgende drei Anforderungskriterien, die an ein solches Instrument gestellt werden, um die von Horvat et al. (2018) genannten Zielsetzungen des strategischen Kompetenzmanagements zu erfüllen (Bimrose et al. 2016; Decius und Schaper 2017; Decius et al.
2018; Galiläer und Wende 2008; Gustavsson 2007; Güth et al. 2018; Illeris 2006; Lundkvist und Gustavsson 2018):

- Handhabbarkeit/vertretbarer Pflegeaufwand: Das Instrument sollte nicht zu komplex gestaltet, im Alltagsgeschäft leicht zu pflegen und zu aktualisieren sein, sowie möglichst geringe Einstiegshürden für neue Anwendende aufweisen.

- Verständlichkeit des Instruments für die Zielgruppe: Das Instrument sollte nicht nur für Personen mit Expertise im Personalbereich oder für die Geschäftsführung verständlich sein, sondern mit Hilfe konkreter Handlungshinweise auch von unteren und mittleren Führungskräften im Produktions- und Logistikbereich eingesetzt werden können.

- Implementierung als datenbankgestütztes System bzw. Anschlussfähigkeit an bestehende Dokumentationen und Systeme: Das Instrument sollte eine technische Umsetzung der Kompetenzerfassung, -dokumentation und -verwaltung zulassen, um praxistauglich $\mathrm{zu}$ sein und im Betrieb nachhaltig zum Einsatz kommen zu können. Dabei sollte eine (ggf. noch nicht datenbankgestützte) Basisversion des Instruments ausreichend flexibel sein, um die Anschlussfähigkeit an bereits im Betrieb etablierte EDV-Systeme ohne großen Umrüstungsaufwand zu ermöglichen.

Die abgeleiteten Kriterien stimmen größtenteils mit den Best-Practice-Merkmalen der Kompetenzmodellierung von Campion et al. (2011) überein, die unter anderem die Berücksichtigung des organisationalen Kontextes, die Nutzung einer betrieblichen Sprache, sowie die Sicherstellung von Akzeptanz und Usability umfassen. Die herausgearbeiteten Bedarfe weisen zudem darauf hin, dass in KMU insbesondere agile Kompetenzmanagementsysteme gefragt sind, die sich unter anderem durch spezifische Anwendungsszenarien, intuitiv zugänglichen Werkzeuge sowie einfache Systeme mit geringem Schulungsbedarf auszeichnen (Kauffeld und Paulsen 2018, S. 45-46). Die Bedeutung des Kriteriums der datenbankgestützten Umsetzung des Kompetenzinstruments steht im Einklang mit den von Kauffeld und Paulsen (2018) genannten Vorteilen IT-gestützter Kompetenzsysteme, die insbesondere die automatische Datenverarbeitung, die unkomplizierte Aufdeckung auch struktureller Kompetenzlücken und verborgener Potenziale sowie den einfachen Abgleich von Ist-Kompetenzen mit unterschiedlichen SollProfilen umfassen (S. 163).

Bei der Bewertung von arbeits- und personaldiagnostischen Instrumenten kommen jedoch in der Regel auch psychometrische Kriterien wie die Reliabilität und die Validität zum Einsatz (Döring und Bortz 2016; Hamborg und Schaper 2018). Inwieweit eine Anwendung psychometrischer Kriterien bei der Kompetenzmodellierung vorteilhaft (Stevens 2013) oder nicht empfehlenswert (Sanchez und Le- 
vine 2009) ist, wird wissenschaftlich kontrovers diskutiert. Unsere Analyse für die Zielgruppe des strategischen Kompetenzmanagements - produzierende KMU - ergab jedoch, dass die praktischen Kriterien klar im Vordergrund stehen. Nichtsdestotrotz wird dieser Aspekt im Rahmen des vorliegenden Beitrags im Abschnitt zu den praktischen Gütekriterien und Nutzungsmöglichkeiten der KMT noch einmal kritisch beleuchtet und Möglichkeiten für eine zukünftige Validierung eruiert. Nachfolgend werden auf Basis der genannten Kriterien drei Kompetenzmessinstrumente verglichen, die für den Einsatz in produzierenden KMU geeignet erscheinen.

\section{Vergleich verschiedener Kompetenzmessinstrumente im Hinblick auf den Einsatz in produzierenden KMU}

In der Praxis und Forschung existiert eine Vielzahl an Kompetenzmessinstrumenten mit verschiedenen Stärken und Schwächen (Erpenbeck und Rosenstiel 2003; Kauffeld und Frerichs 2018; Kauffeld und Paulsen 2018; Kaufhold 2006). Nicht alle dieser Instrumente sind jedoch im Speziellen dafür geeignet, die Bedarfe der Zielgruppe der produzierenden KMU bestmöglich zu erfüllen. Im Folgenden werden in Anlehnung an die Auswahl und Darstellung bei Abel et al. (2016) drei Kompetenzmessinstrumente vorgestellt, die in besonderem Maße für die Anwendung in produzierenden KMU geeignet erscheinen: die Kompetenzmatrix (North et al. 2013), die prozessbezogene Kompetenzmatrix (Hasenau et al. 2013) sowie das ToolPE-Instrumentarium (Galiläer und Wende 2008).

Bei der Kompetenzmatrix von North et al. (2013) findet eine sehr einfache Form der Kompetenzerfassung statt. Den Beschäftigten werden für ihre Arbeit typische Fähigkeiten zugeordnet und anhand von drei Kategorien eingestuft („Kenner“, „Könner“, „Experte“). In der Kompetenzmatrix ist somit bei horizontaler Lesart ersichtlich, wie oft die spezifische Kompetenz in einer Abteilung bzw. im gesamten Unternehmen vorhanden ist. Bei vertikaler Lesart ist erkennbar, welche Kompetenzen eine einzelne Person besitzt. Zwar ist die Kompetenzmatrix einfach anwendbar, transparent und selbsterklärend. Die Kehrseite ist, dass keine hierarchische Verrechnung einzelner Kompetenzen oder eine an Sollwerten orientierte Qualifikationsbedarfsermittlung möglich ist.

Bei der prozessbezogenen Kompetenzmatrix von Hasenau et al. (2013) handelt es sich um ein Instrument, mit dem ein Soll-Ist-Abgleich in Matrixform möglich ist. Auf Gruppen- oder Abteilungsebene kann zudem der kumulierte Bedarf bzw. der kumulierte Bestand abgebildet werden. Die Grundlage für die Entwicklung der Matrix stellt eine Arbeitsprozessanalyse mit fachlichen Interviews, Experten- workshops und Arbeitsbeobachtungen dar. Die Zielgruppenspezifität ist zwar gegeben und auch die abteilungsbezogene Darstellungsform ist positiv zu bewerten. Allerdings wird der Soll-Ist-Abgleich dadurch erschwert, dass keine quantitativ verknüpften Einstufungsschlüssel vorhanden sind, was die Handhabbarkeit der prozessbezogenen Kompetenzmatrix einschränkt.

Das Tool-PE-Instrumentarium von Galiläer und Wende (2008) umfasst drei Teilinstrumente, die zum einen Tätigkeits- und Anforderungsprofile, zum anderen Vorlagen zur Erhebung und Aufbereitung von Qualifikationsanforderungen sowie drittens einen Leitfaden zur Qualifikationsentwicklungsplanung beinhalten. Ähnlich wie bei der prozessbezogenen Kompetenzmatrix werden zu Beginn der Implementierung Fallstudien, Beobachtungen und Interviews durchgeführt. Die festgestellten Teiltätigkeiten können entweder chronologisch oder nach Häufigkeit und Relevanz für die Arbeitsaufgabe abgebildet werden und ergeben ein Anforderungsprofil, mit dem sich die Ist-Einschätzungen abgleichen lassen. Die Anforderungsanalyse verbleibt insgesamt jedoch auf der qualitativen Ebene, sodass eine quantitative Sollwertbestimmung und folglich auch der Soll-IstAbgleich erschwert wird. Positiv hervorzuheben ist die Erhebung formal erworbener Qualifikationen als Ergänzung zu den festgestellten Kompetenzen, wobei allerdings keine Verknüpfung beider Bereiche vorgesehen ist.

Im Hinblick auf die drei aus der Literatur abgeleiteten Kriterien der Handhabbarkeit, der Verständlichkeit sowie der datenbankgestützten Anschlussfähigkeit lässt sich die Verständlichkeit des Instruments für die Zielgruppe der Personen in produzierenden KMU insgesamt als erfüllt bewerten. Die Handhabbarkeit ist ebenfalls größtenteils gegeben und nur geringfügig eingeschränkt. Eine Implementierung als datenbankgestütztes System oder eine Anschlussfähigkeit an bestehende Systeme scheint jedoch nicht vorgesehen zu sein, worauf auch die überwiegend fehlenden Möglichkeiten eines nutzbringenden Soll-ist-Abgleichs mit quantifizierbaren Einstufungsschlüsseln hinweist.

Um den Bedarf der produzierenden KMU zu erfüllen und ein handhabbares, verständliches, konzeptionell fundiertes und im Hinblick auf die IT-Anschlussfähigkeit praxistaugliches Instrument zur Verfügung zu stellen, wird im Folgenden die Kompetenzmanagementtabelle (KMT) vorgestellt. Die Darstellung orientiert sich dabei am Fallbeispiel eines KMU aus der Metallbranche, bei dem die KMT nachhaltig implementiert wurde. 


\section{Das Fallbeispiel: Die alutec metal innovations GmbH \& Co. KG (Metallbranche)}

Bei der alutec metal innovations $\mathrm{GmbH} \& \mathrm{Co} . \mathrm{KG}^{1}$ handelt es sich um ein junges, familiengeführtes Unternehmen mit einer mehr als 30-jährigen Firmengeschichte, welches Aluminiumteile für die Automobilbranche fertigt. Die Kernkompetenz des Unternehmens aus Baden-Württemberg mit ca. 240 Beschäftigten liegt im Bereich des Kaltfließpressens. Jährlich werden auf annähernd $8000 \mathrm{~m}^{2}$ Produktionsfläche ca. 2000t Aluminium umgeformt und anschließend zerspanend bearbeitet und damit ein Umsatz von 25 Mio. $€$ erzielt. Hauptsächlich gefertigt werden Gehäuse, Kühlkörper und Kolben aus Aluminium, die von den alutec-Kunden (d.h., Systemlieferanten der Automobilindustrie) in Steuergeräten, Motorgehäusen, Scheinwerfern oder Bremsen verbaut werden.

In der Produktion sind 50 Fach- und Führungskräfte sowie 150 un- und angelernte Arbeitskräfte beschäftigt. Da sich alutec auf eine Nischentechnologie innerhalb der Aluminiumverarbeitung spezialisiert hat, kommt der Entwicklung von Prozessinnovationen eine hohe Bedeutung zu, um im Markt wettbewerbsfähig zu bleiben. Dies erfordert eine stetige Kompetenzentwicklung der Beschäftigten, insbesondere auch der gering(er) qualifizierten Mitarbeiterinnen und Mitarbeiter in der Produktion, die in Zukunft immer mehr Eigenverantwortung übernehmen müssen. Ein typisches Beispiel ist die Aufgabenerweiterung der Maschinenbedienerinnen und -bediener, die neben der Maschinenbedienung auch Montagetätigkeiten und zunehmend Qualitätskontrollen durchführen, was ein verändertes Kompetenzprofil bei den Beschäftigten erfordert. Um die vorhandenen Kompetenzen sichtbar zu machen, die aktuellen Istwerte mit den zukünftigen Sollanforderungen zu vergleichen und Implikationen für die Weiterbildung im Sinne eines strategischen Kompetenzmanagements abzuleiten, wurde bei alutec die KMT implementiert und deren Anwendung pilotiert (siehe auch Kretz 2018). Dies geschah im Rahmen des vom Bundesministerium für Bildung und Forschung (BMBF) geförderten Verbundforschungsprojekts StraKosphere (,Strategische Kompetenzentwicklung in nichtforschungsintensiven kleinen und mittleren Unternehmen (KMU) des Verarbeitenden Gewerbes“, 2014-2017, vgl. Som und Horvat 2018).

\footnotetext{
${ }^{1}$ Die Darstellung des Unternehmens orientiert sich an Kretz (2018).
}

\section{Konzeptionelle Basis und Entwicklung der KMT}

Die KMT ist bezüglich der Kompetenzmodellierung dem Multiple-Job-Ansatz zuzuordnen (Mansfield 1996). Im Unterschied zum One-Size-Fits-All-Ansatz (bei dem ein Kompetenzmodell für die gesamte Organisation entwickelt wird) und dem Single-Job-Ansatz (bei dem für jede Position ein spezifisches Modell entwickelt wird) handelt es sich um eine Mischform, bei der sowohl übergreifende bzw. „geteilte" als auch jobspezifische Kompetenzen ermittelt werden (Kauffeld und Paulsen 2018, S. 93). Im Vorfeld der Instrumentenentwicklung wurde festgelegt, die KMT hierarchisch zu strukturieren, d.h. die Kompetenzen in Kategorien und Subkategorien zu unterteilen. Dabei erscheint ein Maximum von zwei Hierarchieebenen in Hinblick auf die Anwendbarkeit empfehlenswert zu sein (Campion et al. 2011). Ein Vorteil der Hierarchisierung ist eine Reduktion der Komplexität, die in der Regel durch die Bildung von Kompetenzclustern oder -facetten erreicht wird und zudem die Operationalisierung durch Verhaltensanker umfassen kann (Paulsen und Kauffeld 2019). Die Kompetenzen sollten dabei möglichst aufgabennah formuliert werden (Kauffeld und Paulsen 2018, S. 96).

Das Vorgehen bei der Entwicklung der KMT folgte prinzipiell den drei Schritten von Campion et al. (2011), die wiederum in mehrere untergeordnete Aspekte aufgeteilt sind: (1) Analyse von Kompetenzinformationen (u.a. Durchführung einer grundlegenden Arbeitsanalyse, Betrachtung zukünftiger Arbeitsanforderungen), (2) Organisation und Darstellung der Kompetenzinformationen (u. a. Festlegung des Detailgrades, Definieren von Kompetenzniveaus und Einstufungsschlüsseln), (3) Nutzung der Kompetenzinformationen (u. a. Sicherstellung der Usability und Akzeptanz, Verknüpfung mit bestehenden technischen Systemen).

Ausgangspunkt der Analyse von Kompetenzinformationen für die Instrumentenentwicklung waren mehrere, aufeinander aufbauende Workshops in vier KMU des verarbeitenden Gewerbes, die am Forschungsprojekt StraKosphere beteiligt waren. Neben dem bereits im Rahmen des Fallbeispiels vorgestellten Betrieb - der alutec metal innovations $\mathrm{GmbH} \& \mathrm{Co}$. KG - waren Unternehmen aus der Metall- und Elektroindustrie, der Möbelfertigung sowie des Maschinenund Werkzeugbaus im Projekt vertreten. Die WorkshopAgenda folgte den sechs Prozessschritten für Implementierungsworkshops im Kompetenzmanagement nach Mansfield (1996): (1) laufende und zukünftig antizipierte Veränderungen in der Organisation und der Branche; (2) Grundstock von Hauptverantwortlichkeiten identifizieren; (3) die wichtigsten Aufgaben oder Arbeitsergebnisse für Stelleninhaberinnen und -inhaber; (4) die Leistungskriterien für jede Schlüsselaufgabe; (5) die Basiskompetenzen zur Er- 
füllung der Schlüsselaufgaben; (6) Job-spezifische Verhaltensweisen, an denen sich die Kompetenzen ablesen lassen. Teilnehmende Personen in den Workshops waren jeweils Vertreterinnen und Vertreter der Geschäftsführung, der Produktionsleitung und des Personalmanagements. Die Anzahl der Personen lag dabei je nach KMU bei fünf bis zehn Personen, zuzüglich drei bis fünf Wissenschaftlerinnen und Wissenschaftler (je nach Termin).

In den jeweils zwei einleitenden Workshops pro KMU wurden zunächst die Herausforderungen und Potenziale des jeweiligen Unternehmens identifiziert und analysiert (die folgende Darstellung der Workshop-Inhalte orientiert sich an Güth et al. 2018). Neben einer Diskussion über die wichtigsten Trends in der Branche sowie die sich daraus ergebenden Anforderungen der Zukunft an die Innovationsund Wettbewerbsfähigkeit des jeweiligen KMU sollten die Workshop-Teilnehmenden sich in Kleingruppen außerdem überlegen, weshalb ihr KMU in fünf Jahren zum innovativsten Unternehmen des jeweiligen Bundeslandes gewählt werden sollte (Horvat et al. 2018). Auf der Basis dieser Analyseergebnisse wurden Ziele für die mittel- und langfristige Unternehmensausrichtung abgeleitet (z.B. im Fall von alutec verbesserte Lieferzeiten, weltweite Vernetzung und verstärkte Prozessinnovationen), um die zu entwickelnden Kompetenzen nachhaltig verankern zu können. Aus den strategischen Zielen wurden anschließend die Entwicklungsbedarfe für einzelne betriebliche Funktionsfelder abgeleitet. Als standardisierte Methode kamen Ursache-Wirkungs-Diagramme (sog. Ishikawa-Diagramme) zum Einsatz. Für jedes Funktionsfeld (z. B. im Fall von alutec Montage, Maschinenführung, Kommissionierung etc.) wurden außerdem die Kompetenzanforderungen festgehalten, die zur Erreichung jedes Ziels wichtig sind, wie vertiefte Maschinen- und Produktkenntnisse, Qualitätsbewusstsein und Verantwortungsbereitschaft für Qualität und Sicherheit oder gute Deutschkenntnisse.

Als konzeptionelle Grundlage für die Entwicklung der KMT wurde im Anschluss an die einleitenden Workshops der ,Leitfaden zur qualitativen Personalplanung bei technisch-organisatorischen Innovationen“ (LPI; Sonntag et al. 1999) herangezogen. Hierbei handelt es sich um ein Instrumentarium zur systematischen, detaillierten und prospektiv orientierten Qualifikationsbedarfsermittlung. Der LPI wurde auf der Basis von aufgabenorientierten und handlungstheoretischen Arbeitsanalysekonzepten entwickelt und ermöglicht die Analyse und Bewertung von Qualifikationsanforderungen insbesondere bei industriellen Tätigkeiten, die operative, planerische, diagnostische, koordinierende und personalführende Aufgaben beinhalten (Sonntag et al. 1999). Der LPI für Stelleninhaber umfasst die Erhebung von Informationen und Einschätzungen $\mathrm{zu}$ (1) personalwirtschaftlichen Strukturdaten (z. B. Qualifikation des Stelleninhabers), (2) Aufgaben und Funktionsbereichen (z.B.
Maschinenbedienung und -überwachung), (3) Kommunikations- und Kooperationsanforderungen (z.B. Kooperation in der Arbeitsgruppe), (4) Informationsaufnahme, -verarbeitung und -erzeugung (z.B. Informationserzeugung/ praktisches Handeln), (5) sowie diversen Kenntnissen (z. B. Produkt- und Werkstoffkenntnisse).

In den beschriebenen vier KMU des verarbeitenden Gewerbes wurde der LPI von Führungskräften mittlerer und höherer Hierarchieebenen bearbeitet, jeweils in Hinblick auf verschiedene konkrete Montage- oder Einrichtungstätigkeiten (z. B. im Fall von alutec Maschinenbediener, Maschineneinrichter, Montagearbeiter). Anhand der LPI-Einstufungsschlüssel bewerteten die Führungskräfte die jeweilige Tätigkeit - je nach LPI-Kategorie - beispielsweise in den Dimensionen Häufigkeit der Anforderung (von 0 nie bis 4 täglich) und Kenntnisanforderungen (von 0 keine Kenntnisse erforderlich bis 3 sehr detaillierte Kenntnisse erforderlich; vgl. Sonntag et al. 1999).

Die Auswertung der LPI-Bögen ergab, welche Anforderungskategorien branchenübergreifend für Beschäftigte im produzierenden und verarbeitenden Gewerbe relevant sind. Ein zentrales Ergebnis war dabei, dass ein fachbezogener Kompetenzbereich, der einerseits Wissen/Kenntnisse und andererseits Fertigkeiten/Fähigkeiten gebündelt umfasst, eine zu geringe Granularität für den praktischen Einsatz im Betrieb aufweisen würde. Daher werden beide Bereiche in der KMT unterschieden: Die fachliche Kompetenz bezieht sich auf Fähigkeiten und Fertigkeiten, die kenntnisbezogene Kompetenz auf Wissen und Kenntnisse. Die KMT-Kompetenzbereiche weisen somit größtenteils Überschneidungen, allerdings auch gewisse Unterschiede $\mathrm{zu}$ in der Fachliteratur üblichen Kompetenzschemata auf, wie die Vierteilung in Fach-, Methoden-, Sozial- und Selbstkompetenz (z. B. Kauffeld 2006, oder Schaper 2019a) oder in Knowledge, Skills, Abilities und Other Characteristics (kurz KSAO; z. B. Campion et al. 2011). Während die bei den KSAO vorgenommene Unterscheidung in Wissen und Fertigkeiten/Fähigkeiten ${ }^{2}$ auch in der KMT vorgenommen wird, orientiert sich die methodische Kompetenz und die interpersonelle Kompetenz an den Definitionen von zweien der vier oben genannten Kompetenzbereiche. Die interpersonelle Kompetenz ist dabei größtenteils deckungsgleich mit der Sozialkompetenz, und die methodische Kompetenz entspricht der Methodenkompetenz. Die betriebliche Anforderungsanalyse ergab ferner, dass die Selbstkompetenz für den gewerblichen Bereich in KMU eher eine untergeordnete Rolle spielt. Aus pragmatischen Gründen wurden daher die wenigen Teilkompetenzen, die sich auch der Selbstkompetenz hätten zuordnen lassen können, in den erweiterten, über die Sozi-

\footnotetext{
${ }^{2}$ Fertigkeiten (Skills) und Fähigkeiten (Abilities) werden bei in der KSAO-Unterteilung unterschieden, in der KMT jedoch gemeinsam als Fachkompetenz zusammengefasst.
} 
alkompetenz hinausragenden Kompetenzbereich der interpersonellen Kompetenz eingeordnet. Leistungsbereitschaft - die von Kauffeld und Grote (2019) als Beispiel für die Selbstkompetenz genannt wird - kann beispielsweise auch als Bereitschaft, das Team zu unterstützen, interpretiert und somit als interpersonelle Kompetenz aufgefasst werden.

Die vier grundlegenden KMT-Kompetenzbereiche fachlich, interpersonell, methodisch und kenntnisbezogen können folgendermaßen definiert werden (vgl. Decius et al. 2018; Güth et al. 2018):

- Fachliche Kompetenz umfasst alle Fähigkeiten und Fertigkeiten, die direkt mit Arbeitshandlungen verbunden sind, z. B. Montagefertigkeit, Kontrollieren, Störungen suchen.

- Interpersonelle Kompetenz umfasst angemessenes Verhalten im zwischenmenschlichen Umgang, z. B. Informationen weitergeben, sich mit anderen abstimmen, sich kooperativ verhalten.

- Methodische Kompetenz umfasst alle Sinnes- und Denkleistungen, z.B. Planen, Entscheiden, Informationen wahrnehmen und verarbeiten.

- Kenntnisbezogene Kompetenz umfasst das kognitive Wissen und alle aus dem Gedächtnis abrufbaren Informationen, z. B. Maschinenkenntnisse, Produktkenntnisse, Sicherheitskenntnisse.

\section{Aufbau der KMT und Prozessschritte bei der Implementierung}

Die Prozessschritte bei der Implementierung der KMT folgen dem klassischen Vorgehen bei der Einführung von Kompetenzmanagementsystemen (siehe z.B. Paulsen und Kauffeld 2019) und sollen im Folgenden am Fallbeispiel der bereits vorgestellten alutec metal innovations $\mathrm{GmbH} \&$ Co. KG näher ausgeführt werden.

Der Aufbau der KMT ergibt sich aus den Kompetenzbereichen, Teilkompetenzen, Kompetenzankern - hier wird die erwähnte hierarchische Struktur der KMT deutlich - sowie Einstufungsschlüsseln und der kumulierten Erfüllung der gewichteten Sollwerte (die Darstellung orientiert sich an Decius et al. 2018). Außerdem werden die zusätzlichen Analysebausteine für sprachliche und außerberufliche Kompetenzen vorgestellt. Die KMT als editierbare Excel-Datei mit allen Verrechnungsformeln ist als Online-Zusatzmaterial zu diesem Artikel verfügbar.

\subsection{Kompetenzbereiche, Teilkompetenzen und Kompetenzanker}

Die KMT (vgl. Abb. 1) umfasst wie oben erwähnt die vier Kompetenzbereiche fachlich, interpersonell, methodisch und kenntnisbezogen (der optionale Bereich sprachlich wird gesondert in einem nachfolgenden Kapitel erläutert), die wiederum als Oberkategorien für verschiedene Teilkompetenzen (,Tätigkeitsbeherrschung und Kenntnisse“) dienen. Dem Kompetenzbereich fachlich können beispielsweise die Teilkompetenzen Arbeitsplatz vorbereiten, Montagefertigkeit und Qualitätskontrolle zugeordnet werden.

Jede Teilkompetenz wird durch sog. Kompetenzanker definiert (d.h. Verhaltensindikatoren; Paulsen und Kauffeld 2019; Stevens 2013), die in der KMT hinter dem Namen der Teilkompetenz aufgezählt werden (vgl. Abb. 1). Die Kompetenzanker beschreiben die Teilkompetenz anhand von differenzierteren Aufgaben- bzw. Kompetenzelementen und geben dem Anwender eine Orientierungshilfe, welche Tätigkeiten in der Arbeit für die Kompetenzeinschätzung heranzuziehen sind. Für die bei alutec besonders bedeutsame Teilkompetenz Montagefertigkeit können beispielsweise folgende Anker verwendet werden: Montagematerial und zu montierende Komponenten entnehmen, Montageanleitung aufrufen, Montage durchführen.

Sofern die Kompetenzanker einen unterschiedlichen Grad an Schwierigkeit und Komplexität aufweisen, werden die weniger komplexen Kompetenzanker zuerst genannt. Beispielsweise ist das Aufrufen der Montageanleitung in diesem Beispiel weniger komplex im Vergleich mit der Durchführung der Montage. Diese Systematik folgt dem Aufbau des LPI (Sonntag et al. 1999), an dem sich die KMT orientiert.

Zur Implementierung der KMT wurden betriebsinterne Workshops durchgeführt, an denen überwiegend die gleichen Personen wie bei den Auftaktworkshops teilnahmen. Übergeordnetes Ziel der Workshops war die Berücksichtigung der vier Phasen, die bei der Initiierung von Pilotprojekten im Sinne eines agilen Kompetenzmanagements durchlaufen werden sollten (Kauffeld und Paulsen 2018, S. 48): (1) Informieren und Bereitschaft entwickeln; (2) Planen; (3) Durchführen; (4) Bewerten und Weiterentwickeln.

Im Workshop bei alutec sollten zur Kompetenzermittlung zunächst die aktuellen und zukünftigen Anforderungen an die zu erfassende Tätigkeit - im Fall von alutec die Montagetätigkeit - gesammelt und qualitativ dokumentiert werden. Anschließend wurden für die zu analysierende Montagetätigkeit die relevanten bzw. erforderlichen Teilkompetenzen innerhalb des bestehenden Rasters der vier Kompetenzbereiche festgelegt und in die KMT eingetragen. Dabei sollten sich die Workshop-Teilnehmenden an folgender Leitfrage orientieren: Welche Aufgabenschritte kommen in der Arbeitstätigkeit vor (z.B. Montage, Qualitätskontrolle und Instandhaltung) oder sind für die Aufgabenerfüllung wichtig (z.B. Kooperationsverhalten, Entscheidungsleistungen)? Die einzelnen Teilkompetenzen für die zu erfassende Tätigkeit sollten ein vergleichbares Abstraktionsniveau aufweisen. Ein Negativbeispiel wäre, neben einer umfassenden Teilkompetenz wie Montagefertigkeit einen 


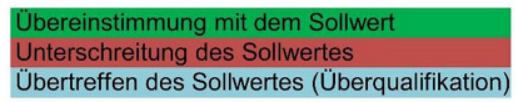

\begin{tabular}{|ll|r|r|r|}
\cline { 3 - 5 } \multicolumn{2}{c|}{} & Person1 & Person2 & Person3 \\
\hline \% Erfüllung des gewichteten Sollwertes: & Fachlich & 100 & 64 & 86 \\
\hline$\%$ Erfüllung des gewichteten Sollwertes: & Interpersonell & 63 & 100 & 75 \\
\hline$\%$ Erfüllung des gewichteten Sollwertes: & Methodisch & 71 & 43 & 43 \\
\hline$\%$ Erfüllung des gewichteten Sollwertes: & Kenntnisbezogen & 100 & 67 & 67 \\
\hline$\%$ Erfüllung des gewichteten Sollwertes: & Sprachlich & 50 & 100 & 100 \\
\hline
\end{tabular}

\begin{tabular}{|c|c|c|c|c|c|c|}
\hline Kompetenz & Tätigkeitsbeherrschung und Kenntnisse & Häufigkeit & $\begin{array}{l}\text { SOLL-Wert } \\
\text { Kenntnisse }\end{array}$ & Person1 & Person2 & Person3 \\
\hline Fachlich & $\begin{array}{l}\text { Arbeitsplatz vorbereiten (z.B. individuell anpassen \& mit Montage- und } \\
\text { Verpackungsmaterial bestücken, Werkzeug auswählen, Bildschirminhalte }\end{array}$ & 2 & \begin{tabular}{|c|c|c|c|c|c|} 
\\
\end{tabular} & 3 & 1 & 2 \\
\hline Fachlich & $\begin{array}{l}\text { Montage (z.B. Montagematerial und zu montierende Komponenten } \\
\text { entnehmen, Montageanleitung aufrufen, Montage durchführen) }\end{array}$ & 3 & 3 & 3 & 1 & 2 \\
\hline Fachlich & $\begin{array}{l}\text { Qualitätskontrolle (z.B. Sichtkontrollen, Messgeräteinsatz, } \\
\text { Funktionsprüfungen, Referenzen abgleichen, Stückliste prüfen) }\end{array}$ & 3 & 3 & 3 & 3 & 3 \\
\hline Interpersonell & $\begin{array}{l}\text { Kooperationsverhalten (z.B. Abstimmung in der Arbeitsgruppe, } \\
\text { eigenverantwortliche und gleichberechtige Arbeitsaufteilung) }\end{array}$ & 1 & 2 & 1 & 2 & 3 \\
\hline Interpersonell & $\begin{array}{l}\text { Kommunikationsverhalten (z.B. Weitergabe von Informationen, } \\
\text { gemeinsames Problemlösen, Kommunikation mit dem Vorgesetzten) }\end{array}$ & 2 & 3 & 2 & 3 & 2 \\
\hline Methodisch & $\begin{array}{l}\text { Informationsaufnahme (z.B. Vergleichen, Übernehmen von } \\
\text { Handlungsanweisungen, Klassifizieren) }\end{array}$ & 2 & 3 & 2 & 1 & 1 \\
\hline Methodisch & $\begin{array}{l}\text { Entscheidungsleistungen (z.B. einfache Entscheidungen zu } \\
\text { Ausführungsalternativen, Entscheidungen über die Arbeitsreihenfolge) }\end{array}$ & 1 & 1 & 1 & 3 & 2 \\
\hline Kenntnisbezogen & $\begin{array}{l}\text { Maschinen- und Anlagenkenntnisse (z.B. Handhabung von Werkzeugen, } \\
\text { Einrichten, Umrüsten) }\end{array}$ & 2 & 2 & 2 & 1 & 1 \\
\hline Kenntnisbezogen & Verfahrenskenntnisse (z.B. Arbeitsabläufe im gesamten Betrieb) & 2 & 1 & 1 & 2 & 2 \\
\hline Sprachlich & Produktion: "Schreiben" (z. B. kurze Mitteilungen verfassen) & 1 & 2 & 1 & 2 & 2 \\
\hline
\end{tabular}

\section{Einstufungsschlüssel für Sollwerte (Anpassung für Istwerte in Klammern)}

0 Hierzu sind keine Kenntnisse / keine Beherrschung erforderlich (vorhanden)

1 Hierzu sind Grundkenntnisse / eine grundlegende Beherrschung erforderlich (vorhanden)

2 Hierzu sind vertiefte Kenntnisse / eine sichere Beherrschung in Standardsituationen

erforderlich (vorhanden)

3 Hierzu sind sehr detaillierte Kenntnisse / eine sichere Beherrschung auch in Ausnahmeund problemhaltigen Situationen erforderlich (vorhanden)
Einstufungsschlüssel für die Häufigkeit der Aufgabe

0 Teilaufgabe kommt in der Arbeit nicht vor

1 Teilaufgabe kommt nur in Ausnahmefällen vor

2 Teilaufgabe gehört zum festen Aufgabenbestand, kommt

2 aber nur in unregelmäßigen Abständen vor

3 Täglich zu verrichtende bzw. regelmäßig vorkommende

Teilaufgabe

Abb. 1 Die Kompetenzmanagementtabelle (KMT), beispielhaft ausgefüllt für eine Montagetätigkeit

sehr eingeschränkten Tätigkeitsaspekt wie Schraubengrößen erkennen anzugeben. Letzteres wäre vielmehr als verhaltensbezogener Kompetenzanker einzuordnen bzw. zu benennen. Außerdem sollten sich die Teilkompetenzen möglichst trennscharf voneinander abgrenzen lassen. Ein Negativbeispiel hierzu wäre, Beobachten des Produktionsablaufes und Überwachen des Betriebszustandes als getrennte Teilkompetenzen zu benennen. Die Teilkompetenzen inklusive ihrer Anker waren in einem weiteren Schritt hinsichtlich verschiedener Aspekte einzustufen bzw. zu bewerten.

\subsection{Einstufungsschlüssel}

Für die Bewertung der Kenntnis- bzw. Fertigkeitstiefe wurden Einstufungsschlüssel entwickelt (vgl. Mansfield 1996), um den Nutzerinnen und Nutzern der KMT (im Fall von alutec Personen der Personalabteilung, Betriebsleiter, Bereichsleiter und Schichtführer) die Einstufung der Teilkompetenzen für den jeweiligen Arbeitsbereich (Sollwerte) und die Einschätzung der jeweiligen Beschäftigten (Istwerte) zu erleichtern. Die Einstufungsschlüssel für Soll- und Istwerte unterscheiden sich sprachlich darin, ob von erforderlichen (Soll) oder vorhandenen (Ist) Kenntnissen bzw. Fertigkei- ten die Rede ist. Außerdem wird für jede Teilkompetenz die Häufigkeit des Auftretens bestimmt, wozu ebenfalls ein entsprechender Einstufungsschlüssel entwickelt wurde.

\subsubsection{Kenntnistiefe (Soll- und Istwerte)}

Für jede Teilkompetenz wurde für jeden Arbeitsplatz ein betriebsspezifischer Sollwert festgelegt und in die KMT eingetragen. Dies sollte, wenn möglich, nicht nur durch eine Person oder Stelle, sondern durch mehrere Verantwortliche im Konsens und nach einer Diskussion und Abstimmung über die jeweiligen Sollwerte sowohl von Seiten der Produktionsleitung als auch der Personalleitung vorgenommen werden. Hierzu bietet sich, wie bei alutec geschehen, die Durchführung eines weiteren Workshops der beteiligten Personen an. Wichtig ist dabei, dass der Sollwert sich ausschließlich auf die Anforderungen bei der betrachteten Tätigkeit, in diesem Beispiel eine Montagetätigkeit, und nicht auf die Kenntnisse bzw. das Können der aktuell ausführenden Arbeitskraft bezieht. Dabei wird in der KMT folgender Einstufungsschlüssel zu Kenntnistiefe/ Beherrschungsgrad mit einer Skalierung von 0 bis 3 verwendet. 


\section{Kenntnistiefe/Beherrschungsgrad (SOLL):}

(0) hierzu sind keine Kenntnisse/keine Beherrschung erforderlich

(1) hierzu sind Grundkenntnisse/eine grundlegende Beherrschung erforderlich

(2) hierzu sind vertiefte Kenntnisse/eine sichere Beherrschung in Standardsituationen erforderlich

(3) hierzu sind sehr detaillierte Kenntnisse/eine sichere Beherrschung auch in Ausnahme- und problemhaltigen Situationen erforderlich

Für die sich später ggf. anschließende Qualifizierung könnten die Sollwerte folgendermaßen interpretiert werden:

(1) grundlegende Einweisung erforderlich

(2) zusätzliche Erfahrungen oder Unterweisungsschritte sind bei der Erlernung erforderlich

(3) komplexere und umfangreichere Vermittlung von Kenntnissen und Vorgehensweisen unter Berücksichtigung besonderer Bedingungen sind erforderlich

Je nach Anwendungszweck der KMT sollten die betrieblichen Anwenderinnen und Anwender vorab die Frage klären, ob die Sollwerte für die Anforderungen der aktuellen Tätigkeit zu bestimmen sind, oder die in Zukunft zu erwartenden Anforderungen bzw. erforderlichen Kompetenzen einer Tätigkeit - vor dem Hintergrund bestimmter Veränderungen oder Entwicklungstrends in der Produktion antizipiert bzw. prognostiziert werden sollen (bei alutec war Letzteres der Fall). Dies erleichtert eine effiziente Diskussion in den betriebsinternen Workshops. Der Fokus auf aktuelle Sollwerte ist handfester und zeigt die gegenwärtigen Kompetenzentwicklungsbedarfe auf. Ein Fokus auf die zukünftigen Bedarfe kann für den Betrieb wertvoller sein, setzt aber konkrete Vorstellungen über die Entwicklung der zukünftigen Anforderungen voraus. In jedem Fall sollten sich die Sollwerte realistisch und differenziert aus den betrieblichen Anforderungen ergeben; verallgemeinerte „Wunschwerte“, bei denen in nahezu allen Teilkompetenzen der höchste Sollwert gewählt wird, sind wenig zielführend bzw. praktikabel.

Nach der Festlegung der Sollwerte wurde in einem weiteren Schritt für jede beschäftigte Person eingestuft, wie gut er oder sie jede Teilkompetenz erfüllt (Istwerte). Für die Einstufung der Istwerte bei alutec wurde der gleiche Einstufungsschlüssel wie für den Sollwert verwendet, lediglich mit angepassten Formulierungen.

\section{Kenntnistiefe/Beherrschungsgrad (IST):}

(0) keine Kenntnisse/keine Beherrschung vorhanden

(1) Grundkenntnisse/eine grundlegende Beherrschung vorhanden

(2) vertiefte Kenntnisse/eine sichere Beherrschung in Standardsituationen vorhanden

(3) sehr detaillierte Kenntnisse/eine sichere Beherrschung auch in Ausnahme- und problemhaltigen Situationen vorhanden

In der KMT werden im Anschluss an die Soll- und Istwert-Bestimmung die Soll-Istwert-Differenzen für alle bewerteten Teilkompetenzen pro Mitarbeiterin und Mitarbeiter als detaillierte Auswertung der Kompetenzbedarfe dargestellt. Außerdem wird eine aggregierte Auswertung zu den Kompetenzbereichen angeboten, für die berechnet wird, wieviel Prozent der Sollanforderungen von jedem Mitarbeiter pro Bereich bereits erfüllt bzw. beherrscht werden. Weist z. B. Person 1 für die Teilkompetenz Montagefertigkeit eine sichere Beherrschung in Standardsituationen auf, wird in die entsprechende Tabellenzelle eine 2 eingetragen. Geht man nun davon aus, dass zuvor für den Sollwert der Teilkompetenz Montage eine 3 (sichere Beherrschung auch in Ausnahme- und problemhaltigen Situationen) vermerkt wurde, so ergibt sich eine Abweichung von -1 . Der Mitarbeiter unterschreitet somit den geforderten Sollwert für die Kompetenzbeherrschung. Dies lässt sich in der KMT durch die Färbung der Zelle ablesen. Rote Zellen signalisieren eine Unterschreitung des Sollwertes, grüne Zellen eine Übereinstimmung mit dem Sollwert. Ein Übertreffen des Sollwertes (Überqualifikation) wird blau hervorgehoben.

Die Einstufung des Sollwertes sollte in der Regel von der Führungskraft der Mitarbeiterin/des Mitarbeiters, deren/ dessen Qualifikationsstand bzw. Kompetenzentwicklungsbedarf anhand der KMT bestimmt wird, vorgenommen werden. Zur Unterstützung der Zuverlässigkeit und Qualität dieser Einstufungen ist zu empfehlen, dass zu Beginn der KMT-Nutzung im Führungskreis über die Einschätzungen diskutiert und eine gegenseitige Abstimmung erfolgen sollte. Ziel ist es, dass betriebsintern ein gemeinsames mentales Modell über die bei den Kompetenzeinschätzungen zu berücksichtigenden Aspekte entstehen kann. Außerdem sollten die Führungskräfte zuvor in den Umgang mit der KMT eingewiesen werden, um Fehlbewertungen zu vermeiden und die Arbeit mit dem Instrument im Betriebsalltag zu erleichtern. Jede Mitarbeiterin und jeder Mitarbeiter soll unabhängig von anderen Beschäftigten eingeschätzt werden sowie unabhängig vom Sollwert. Es empfiehlt sich, die zuvor definierten Sollwerte während der Einstufung der Mitarbeiter auszublenden, damit keine fälschliche Orientierung an den Sollwerten erfolgt. 


\subsubsection{Häufigkeit der Aufgabe}

In der KMT wird neben dem Sollwert und den Istwerten der Beschäftigten außerdem angegeben, wie häufig die jeweilige Teilkompetenz in der Arbeit benötigt wird. Dabei wird ein Einstufungsschlüssel mit einer Skalierung von 0 bis 3 verwendet:

(0) Teilaufgabe bzw. Teilkompetenz kommt in der Arbeit nicht vor

(1) Teilaufgabe bzw. Teilkompetenz kommt nur in Ausnahmefällen vor

(2) Teilaufgabe bzw. Teilkompetenz gehört zum festen Aufgabenbestand, kommt aber nur in unregelmäßigen Abständen vor

(3) täglich zu verrichtende Teilaufgabe bzw. regelmäßig vorkommende Teilkompetenz

Bei der Festlegung der Häufigkeitswerte ist ähnlich zu verfahren wie bei den Sollwerten zur Beherrschung einer Teilkompetenz. Auch hierbei sollte vorab Klarheit darüber herrschen, ob die aktuelle oder zukünftige Häufigkeit einer Anforderung bzw. Teilkompetenz betrachtet werden soll. Bei der Einschätzung der aktuellen Häufigkeit, wie oft eine Teilkompetenz in der Arbeitstätigkeit benötigt wird, können (wie bei alutec) Erfahrungs- und Beobachtungswerte von Schichtführern und Gruppenverantwortlichen herangezogen werden. Wenn für mehrere Arbeitsbereiche die gleiche KMT-Struktur verwendet wird, kann es sinnvoll sein, nicht vorkommende Teilkompetenzen in einem einzelnen Bereich auf ,null“ zu setzen, anstatt sie aus der KMT zu entfernen.

\subsection{Erfüllung der gewichteten Sollwerte}

Mithilfe der gewichteten Sollwerte ist es möglich, innerhalb der KMT für jede Person abzulesen, zu wie viel Prozent diese Person die betrieblichen Anforderungen in einem Kompetenzbereich erfüllt. Der Sollwert wird durch eine Multiplikation mit dem Häufigkeitswert gewichtet. Hintergrund der Gewichtung des Sollwertes mit der Einstufung der Häufigkeit ist, dass täglich stattfindende Aufgaben gegenüber selteneren Aufgaben eine größere Bedeutung haben und sich dies auch in der KMT widerspiegeln soll. Dazu wird der Sollwert mit dem Häufigkeitswert multipliziert. Das Ergebnis der Multiplikation geht automatisch in die Berechnung des prozentualen Erfüllungswertes ein.

Um den prozentualen Erfüllungsgrad festzustellen, wird in der KMT für jede Person die Abweichung der (mit der Häufigkeit gewichteten) Istwerte von den (mit der Häufigkeit gewichteten) Sollwerten berechnet. Der Erfüllungsgrad wird in der KMT nicht für jede Teilkompetenz einzeln, sondern für jeden Kompetenzbereich dargestellt. Für den
Kompetenzbereich interpersonell (am Beispiel einer Montagetätigkeit, vgl. Abb. 1) ergibt sich der Gesamtwert der prozentualen Erfüllung aus den durchschnittlichen Erfüllungsgraden der beiden Teilkompetenzen Kooperationsverhalten und Kommunikationsverhalten. Beträgt dieser kumulierte Erfüllungswert - wie im Beispiel zu sehen -63 bei Person 1, bedeutet dies, dass Person 1 die innerbetrieblich festgelegten Sollwerte unter Berücksichtigung der Häufigkeit, wie oft die enthaltenen Teilkompetenzen in der Aufgabe vorkommen, zu $63 \%$ erfüllt.

Die KMT bietet die Möglichkeit auf einer aggregierten Ebene, die prozentuale Erfüllung der gesamten Kompetenzbereiche jedes Beschäftigten auf einen Blick abzulesen. Beträgt der Kumulationswert bei fachlicher Kompetenz beispielsweise 73, bedeutet dies, dass Person 1 die Sollwerte der fachlichen Kompetenz zu $73 \%$ erfüllt. Durch die Gewichtung der Sollwerte mit der Häufigkeit können folgende Beispiele von benötigten Teilkompetenzen adäquat in der KMT abgebildet werden (Decius et al. 2018):

- Eine Aufgabe in der Produktion mit geringer Häufigkeit, aber einer hohen vorausgesetzten Kenntnistiefe, wäre z. B. der Umgang mit Notsituationen (Brandfall etc.).

- Umgekehrt ist die Maschinenbedienung eine häufig vorkommende Aufgabe, die als Routinetätigkeit eine lediglich geringe Kenntnistiefe benötigt.

- Wenig anspruchsvolle und zugleich seltene Tätigkeiten wären z. B. das Ausfüllen von Dokumenten und das Führen von Listen.

- Die von einem Instandhalter ausgeführten Wartungs- und Reparaturarbeiten sind für diesen in der Regel eine tägliche Aufgabe, die zudem ein hohes Kenntnisniveau voraussetzt.

\subsection{Zusatzanalysetool für sprachliche Kompetenzen}

Neben den vier Kompetenzbereichen fachlich, interpersonell, methodisch und kenntnisbezogen bietet die KMT ebenfalls die Möglichkeit, für Beschäftigte im Produktionsbereich relevante sprachliche Teilkompetenzen abzubilden bzw. zu erfassen und zu bewerten. Für Produktionsbetriebe wie alutec, die Mitarbeiterinnen und Mitarbeiter aus vielen verschiedenen Ländern beschäftigen, ist dies im Sinne einer guten gemeinsamen Verständigung sehr wichtig. Standardmäßig sind im Kompetenzbereich sprachlich folgende fünf Teilkompetenzen enthalten (vgl. Decius et al. 2018):

(1) Produktion: „Sprechen - individuell“ (z. B. sich verständlich ausdrücken, Informationen in eigenen Worten ausdrücken, in einem natürlichen Redefluss sprechen, Fachausdrücke benutzen)

(2) Produktion: „Sprechen - interaktiv“ (z.B. zielorientiert kooperieren) 
(3) Produktion: „Schreiben“ (z.B. kurze Mitteilungen verfassen)

(4) Rezeption: „Hören“ (z.B. Anweisungen verstehen, Vorträgen in Schulungen folgen können)

(5) Rezeption: „Lesen“ (z.B. schriftliche Mitteilungen wie technische Instruktionen verstehen)

Zur Beurteilung der sprachlichen Kompetenzen hält die KMT für jede Teilkompetenz einen ausführlichen Einstufungsschlüssel bereit (siehe Online-Zusatzmaterial zu diesem Artikel). Die Einstufungsschlüssel orientieren sich am Gemeinsamen europäischen Referenzrahmen für Sprachen (vgl. Trim et al. 2001). In der KMT wird für die Einstufung eine Skalierung von 0 bis 3 verwendet. Jede Stufe ist in den Einstufungsschlüsseln mit ausführlichen Verhaltensbeispielen beschrieben, um die einzelnen Stufen voneinander abgrenzen zu können und eine Beurteilung der Beschäftigten zu erleichtern. Der Einstufungsschlüssel für Sprechenindividuell sieht beispielsweise folgendermaßen aus:

(1) Mitarbeiter spricht selbst kein Deutsch und verständigt sich auf Umwegen (z. B. über Gesten)

(2) Mitarbeiter benutzt sehr kurze, isolierte Äußerungen, macht dabei sehr viele Pausen (Fehler sind häufig); der Wortschatz ist auf alltagssprachliche Ausdrücke beschränkt

(3) Mitarbeiter versucht Redebeiträge größtenteils - etwas fehleranfällig und unstrukturiert - selbst $\mathrm{zu}$ formulieren und kommt dabei öfters ins Stocken; er/sie verfügt über einen elementaren allgemeinen Wortschatz und kann Formulierungen variieren, ohne allzu häufige Wiederholungen

(4) Mitarbeiter kann eigene Bedürfnisse und nicht alltägliche arbeitsbezogene Sachverhalte eigenständig und relativ fehlerfrei für andere nachvollziehbar darlegen; er/sie verfügt über einen reichen Wortschatz (kann auch mit Fachausdrücken umgehen)

\subsection{Zusatzanalysetool für außerberufliche Kompetenzen}

Abseits der Kompetenzen, die sich aus den beruflichen Aufgaben ergeben, können in der KMT auch solche Kompetenzen dargestellt werden, die die Beschäftigten im außerberuflichen und ehrenamtlichen Bereich erworben haben. Dabei wird davon ausgegangen, dass diese außerberuflichen Kompetenzen mit den beruflichen Kompetenzen verbunden sind und daher auch eine Relevanz für die Arbeitstätigkeit besitzen. Beispiele wären handwerkliche Fertigkeiten aus dem Hobbybereich oder Führungskompetenzen aus Ehrenämtern in Vereinen. In der KMT werden dabei 14 Tätigkeitsbereiche unterschieden, z.B. technisch/handwerklich tätig sein, in und mit Gruppen arbeiten, im Gemeinwesen/Politik tätig sein (für die vollständige Auflistung siehe Decius et al. 2018). Die Tätigkeitsbereiche der KMT basieren dabei auf den Darstellungen aus zwei bereits bestehenden Kompetenzanalysetools: auf dem AiKomPass aus dem Projekt $A i$ Ko - Anerkennung informell erworbener Kompetenzen (Fischer et al. 2015) sowie teilweise auf dem KMU-Kompetenzbuch (Heil et al. 2011). Ein für alutec besonders relevantes Beispiel aus dem Bereich technisch/handwerklich tätig sein ist: mit Materialien wie Stein, Beton, Holz, usw. etwas bauen, etwas herstellen bzw. restaurieren (Bsp. Möbel restaurieren). Personen, die handwerklich erfahren sind, können innerhalb des Betriebs auch außerhalb der Maschinenbedienung vielseitig eingesetzt werden und eventuell für die Werkzeugfertigung qualifiziert werden.

Die einzelnen Tätigkeiten können den vier Kompetenzbereichen fachlich, interpersonell, methodisch und kenntnisbezogen zugeordnet werden, um den Zusammenhang der außerberuflichen Kompetenzbereiche mit den Arbeitstätigkeiten zu verdeutlichen. Zur Unterstützung der Benutzerfreundlichkeit der KMT können direkte Verlinkungen zwischen den Einstufungen zu den beruflichen und außerberuflichen Kompetenzbereichen eingefügt werden (z.B. zwischen dem Tätigkeitsbereich Wissen vermitteln/ weitergeben/prüfen und der beruflichen Teilkompetenz Anlernen neuer Mitarbeiter/Mitarbeiterinnen).

Es ist davon auszugehen, dass eine Tätigkeit im außerberuflichen Bereich insbesondere dann berufsrelevant sein könnte, wenn sie regelmäßig (Häufigkeit) und auf einem entsprechenden Niveau ausgeübt wird. Die Häufigkeit wird mit dem folgenden Einstufungsschlüssel auf einer Skala von 0 bis 3 bewertet:

(0) Tätigkeit wird in der Freizeit nicht ausgeübt

(1) Tätigkeit wird in der Freizeit gelegentlich ausgeübt

(2) Tätigkeit wird in der Freizeit öfter ausgeübt

(3) Tätigkeit wird in der Freizeit regelmäßig ausgeübt

Das Niveau wird auf einer Skala von 0 bis 4 eingestuft:

(0) Mitarbeiter/-in übt die Tätigkeit nicht aus

(1) Mitarbeiter/-in kann es mit Unterstützung anderer tun

(2) Mitarbeiter/-in kann es selbstständig, ohne Unterstützung anderer tun

(3) Mitarbeiter/-in kann es selbstständig und in neuen $\mathrm{Zu}$ sammenhängen tun

(4) Mitarbeiter/-in kann es selbstständig und in neuen $\mathrm{Zu}$ sammenhängen tun, bewerten und anderen beibringen

Novizinnen und Novizen auf einem Gebiet können die Tätigkeit beispielsweise zunächst nur mit der Unterstützung anderer ausüben, Erfahrene dagegen auch selbständig, Expertinnen und Experten sogar andere Novizinnen und Novi- 
zen anleiten. In der KMT wird die Bewertung der außerberuflichen Kompetenzen anhand des Produkts der Einstufungen für die Häufigkeit sowie für das Niveau der Ausübung gebildet. Ähnlich wie bei den beruflichen Kompetenzbereichen ist auch hier eine kumulierte Darstellung möglich. Sofern ein Nachweis über die außerberufliche Kompetenz vorhanden ist und vom Mitarbeiter bzw. der Mitarbeiterin vorgelegt wurde, kann dies zu Dokumentationszwecken in der KMT vermerkt werden. Dazu existiert der folgende zweistufige Einstufungsschlüssel:

\section{(0) Nachweis nicht vorhanden}

(1) Nachweis vorhanden

Während die beruflichen Kompetenzen der Mitarbeiterinnen und Mitarbeiter von ihren Führungskräften oder zuständigen Personalfachkräften eingeschätzt werden, ist für die Erfassung und Dokumentation der außerberuflichen Kompetenzen die aktive Mitarbeit der Beschäftigten nötig, da nur sie freiwillig Auskunft über ihre Aktivitäten im außerberuflichen Bereich geben können (insbesondere bei einer Einführung dieses Zusatztools sollte, wie auch bei der Implementierung der gesamten KMT, der Betriebsrat einbezogen werden, wie es auch bei alutec der Fall war). Zur Erfassung außerberuflicher Kompetenzen bietet sich beispielsweise das Mitarbeiterentwicklungsgespräch an. Die Führungskraft sollte den Mitarbeiter bzw. die Mitarbeiterin dazu vorab informieren, wozu die Angaben über die auBerberuflichen Kompetenzen genutzt werden und welche beruflichen Weiterbildungsmöglichkeiten sich dadurch für den Mitarbeiter ergeben.

\section{Praktische Gütekriterien und Nutzungsmöglichkeiten der KMT}

\subsection{Gütekriterien}

Die KMT stellt ein praxistaugliches Instrument zur Kompetenzbedarfsermittlung und -planung in KMU dar. Dabei erfüllt die KMT die eingangs erwähnten Kriterien, die KMU an Kompetenzmanagementinstrumente stellen:

- Handhabbarkeit/vertretbarer Pflegeaufwand: Die KMT ist übersichtlich gestaltet und erfordert im Alltagsgeschäft nur einen geringen Aktualisierungsaufwand. Die benutzerfreundliche Darstellung ermöglicht eine hohe praktische Handhabbarkeit, die sich durch eine datenbankgestützte Umsetzung weiter erhöhen lässt (siehe unten).

- Verständlichkeit des Instruments für die Zielgruppe: Dank des vorliegenden Implementierungsleitfadens und der Nutzungshinweise (vgl. Abschn. 6, ausführlich bei
Decius et al. 2018) sind die Einstiegshürden für neue Anwendende der KMT gering. So können auch untere und mittlere Führungskräfte im Produktions- und Logistikbereich nach einer Einweisung bzw. einem gemeinsamen Workshop die KMT im Alltagsgeschäft nutzen.

- Implementierung als datenbankgestütztes System bzw. Anschlussfähigkeit an bestehende Dokumentationen und Systeme: Die KMT liegt in der Basisversion als editierbare Datei in Microsoft Excel vor, um eine möglichst große plattformunabhängige Reichweite $\mathrm{zu}$ erzielen (siehe Online-Zusatzmaterial $\mathrm{zu}$ diesem Artikel). Die Basisversion kann datenbankgestützt beispielsweise in Microsoft Access umgesetzt werden (siehe Fallbeispiel von Kaiser und Kaiser 2018) oder - wie im Fall von alutec - in ein im Betrieb bereits vorhandenes System integriert werden (siehe Kretz 2018). Bei einer IT-Umsetzung der KMT kann mit einer Erhöhung der Effizienz, reduzierten Kosten, einer Verminderung von administrativen Hürden, der Unterstützung von Planungen im Personalmanagement und der Aufwertung der Rolle des Personalmanagements im Betrieb gerechnet werden (Kauffeld und Paulsen 2018, S. 162). Ein BestPractice-Beispiel für eine solche Umsetzung ist das für die Bedarfe des Handwerks entwickelte Kompetenz-Navi welches beispielsweise zur Bedarfsanalyse von internen Trainings und der Zusammenstellung von Lern-Tandems genutzt werden kann (Kortsch et al. 2018).

Wie bereits einleitend angesprochen, wird wissenschaftlich kontrovers diskutiert, inwieweit psychometrische Kriterien zur Evaluation von Kompetenzmanagementsystemen zum Einsatz kommen sollten (Sanchez und Levine 2009; Stevens 2013). Die praxisnahe Entwicklungsweise der KMT unter Einbezug verschiedener Expertinnen und Experten aus Wissenschaft und Praxis in den betrieblichen Workshops legt eine hohe inhaltliche Validität nahe (Moosbrugger und Kelava 2012). Durch die Ableitung der Teilkompetenzen und der Kompetenzanker aus den Tätigkeitsprofilen ist eine hohe Augenscheinvalidität gegeben, die die Akzeptanz der KMT bei den Anwendenden stärken sollte (Döring und Bortz 2016). Weitere Validitätsarten, wie die Konstruktvalidität und die Kriteriumsvalidität (Moosbrugger \& Kelava) sind in zukünftigen Studien noch zu untersuchen. Im aktuellen Zustand kann die KMT gemäß den wissenschaftlichen Konventionen somit nicht als vollständig validiertes Instrument betrachtet werden. In Hinblick auf zukünftige Validierungsbemühungen dürfte aus Praxissicht insbesondere interessant sein, inwieweit die erhobenen Kompetenzen mit Leistungskriterien korrelieren - auch wenn eine Messung dieser Kriterien aufgrund der in der betrieblichen Arbeitsumgebung vorhandenen Störvariablen schwierig ist. 


\subsection{Nutzungsmöglichkeiten}

Insgesamt bietet die KMT verschiedene Nutzungsmöglichkeiten für den Einsatz bei Produktionsbeschäftigten in KMU. Aus einer strukturellen Perspektive können strategische, für die KMU auf betrieblicher Ebene bedeutsame Kompetenzdefizite aufgedeckt werden, die durch Schulungen oder arbeitsintegrierte Lernformate behoben werden könnten (Kauffeld und Paulsen 2018, S. 124). Aus einer individuellen Perspektive können die Führungskräfte oder auch die Beschäftigten selbst (ggf. im Austausch mit Führungskräften) personenbezogene Lernpotenziale identifizieren. Bei der Ergebnisdarstellung der Kompetenzen innerhalb der KMT kann der Fokus zudem auf zwei Ebenen gelegt werden, die der strukturellen und der individuellen Perspektive entsprechen (siehe auch Decius et al. 2018; Güth et al. 2018):

- Zum einen können die Qualifizierungsbedarfe für die einzelnen Teilkompetenzen im Fokus stehen: Hierzu ist eine horizontale Betrachtungsweise der Auswertungen erforderlich. Die Betrachtung pro Zeile in der KMT (Teilkompetenz) über mehrere Mitarbeiterinnen und Mitarbeiter für eine Tätigkeit bzw. einen Bereich ermöglicht den Vergleich, wie die Teilkompetenz bei den einzelnen Beschäftigten ausgeprägt ist und ob hier aus gesamtbetrieblicher Sicht ein Defizit besteht (strukturelle Perspektive). Dies ist insbesondere notwendig, wenn durch altersbedingte personelle Wechsel Kompetenzträger aus dem Unternehmen ausscheiden und damit eine bisher ausreichend vorhandene Teilkompetenz bei den verbleibenden Beschäftigten nicht mehr zur Verfügung steht. Außerdem lässt sich durch die Darstellung der prozentualen Sollwerterfüllung horizontal ablesen, welcher Mitarbeiter bzw. welche Mitarbeiterin in jedem der Kompetenzbereiche den Anforderungen am besten gerecht wird.

- Zum anderen können die Qualifikationsbedarfe der einzelnen Beschäftigten im Fokus stehen: Hierzu wird eine vertikale Betrachtungsweise der Kompetenzeinstufungen verfolgt, bei der die Einstufungen zu allen Teilkompetenzen eines Beschäftigten untereinander ablesbar sind. Durch die farblichen Markierungen (vgl. Abb. 1) fallen Unterschreitungen des Sollwerts auf, sodass Schulungsbedarfe bei den jeweiligen Beschäftigten direkt erkannt werden können. Außerdem kann für jeden Beschäftigten die kumulierte Erfüllung der gewichteten Sollwerte für jeden Kompetenzbereich abgelesen werden, beispielsweise ein Gesamtwert über alle fachlichen oder alle methodischen Teilkompetenzen. So können die KMTAnwendenden erkennen, bei welchen Kompetenzen der einzelne Beschäftigte Stärken oder Schwächen aufweist (individuelle Perspektive).
Die KMT kann den Beschäftigten beispielsweise im Rahmen von Mitarbeiterentwicklungsgesprächen vorgestellt und erläutert werden. Hierbei können auch die vorgenommenen Kompetenzeinschätzungen für den jeweiligen Mitarbeiter bzw. die Mitarbeiterin besprochen werden. Sofern die Beschäftigten bereits mit der KMT vertraut sind, können sie im Vorfeld des Mitarbeiterentwicklungsgesprächs bereits eine Selbsteinschätzung ihrer Kompetenzen mittels der KMT vornehmen, die im Gespräch mit der Fremdeinschätzung durch die Führungskräfte abgeglichen werden kann. Dieses partizipative Vorgehen trägt zu einer höheren Akzeptanz der KMT auf Beschäftigtenseite bei, stärkt die Reflexion der eigenen Kompetenzen der Beschäftigten, und ermöglicht den Führungskräften wertvolle Rückmeldungen zum Einsatz der KMT.

Insgesamt lassen sich mithilfe der KMT drei wichtige Ziele der Personalarbeit in KMU erreichen (Güth et al. 2018): Die KMT dient der Qualifikations- und Bedarfsermittlung, sie kann die Grundlage für die Einarbeitung neuer Mitarbeiter bilden, sowie die Basis für Mitarbeiterbzw. Entwicklungsgespräche darstellen. Weitere nachgeordnete Ziele sind außerdem die Verwendung zu Zwecken der Einsatz-, Urlaubs- und Vertretungsplanung sowie ein insgesamt verbesserter Umgang mit Fluktuation, dem Strukturwandel sowie der Internationalisierung von Geschäftsprozessen. Die KMT kann außerdem gemäß den Bedarfen einzelner KMU individuell angepasst werden, beispielsweise durch Ergänzung von Einstufungskategorien wie „Bedeutung für Qualität und Sicherheit“ und „Schwierigkeit des Lernprozesses“ (für ein Umsetzungsbeispiel siehe Kaiser und Kaiser 2018).

\subsection{Pflege und Aktualisierung der KMT}

Im Sinne eines agilen Kompetenzmanagements (Kauffeld und Paulsen 2018, S. 45) benötigen Kompetenzmanagementinstrumente eine stetige Aktualisierung, um den sich verändernden betrieblichen Bedarfen gerecht zu werden. Der Multiple-Job-Ansatz (Mansfield 1996) der KMT ist prädestiniert für entsprechende Anpassungen und Erweiterungen des Instruments. Insbesondere die Istwert-Einstufungen der Beschäftigten sollten in der KMT stets aktuell gehalten werden. Ist dies im Tagesgeschäft nicht möglich, kann die Neueinstufung aller oder eines Teils der Beschäftigten auch in Intervallen erfolgen, beispielsweise einmal jährlich (Decius et al. 2018; Güth et al. 2018). Da sich die betrieblichen Kompetenzanforderungen über die Zeit ändern, sollten auch die Sollwerte, Kompetenzanker und Teilkompetenzen der KMT in regelmäßigen, für den jeweiligen Betrieb sinnvoll festgelegten Intervallen angepasst werden. Je nach Dynamik der Branche sind Intervalle von einem Jahr, zwei Jahren oder bis zu fünf Jahren zu empfehlen. Eine Anpassung der KMT kann z.B. in Strategie- 
und Planungsworkshops unter Einbezug aller betrieblichen Gruppen (von der Geschäftsführungsebene über die mittleren Führungskräfte und das Personalmanagement bis zu den ausführenden Beschäftigten in der Produktion). Die intervallbasierte Pflege der KMT ähnelt damit einer reduzierten Form der erstmaligen Implementierung, wobei eine datenbankgestützte Anbindung der KMT den Aufwand reduziert. Orientierung geben bei der Aktualisierung kann dabei - neben dem erwähnten agilen Kompetenzmanagement - der dynamische Ansatz des strategischen Kompetenzmanagements (Horvat et al. 2018). Der Ansatz umfasst die folgenden sieben Handlungsschritte: Zur strategisch-organisatorischen Ebene gehören (1) die Analyse und Priorisierung der zukünftigen betrieblichen Trends sowie die Ableitung der strategischen Unternehmensziele, (2) die Priorisierung der abgeleiteten Ziele, (3) die Identifizierung von Veränderungs- und Anpassungsbedarfen in Arbeitsstrukturen, Prozessen und organisatorischen Rahmenbedingungen, sowie (4) die Analyse, Aufstellung bzw. Anpassung von Kompetenz-Sollprofilen. Zur individuellen Ebene gehören (5) die Kompetenzanalyse und Ableitung von Handlungsbedarfen, (6) die Auswahl und Umsetzung von Maßnahmen zur Kompetenzentwicklung, sowie (7) die Evaluation der umgesetzten Maßnahmen und eventuelle Anpassung.

\section{Ausblick und Fazit}

Mit der KMT steht ein für den Einsatz in KMU optimiertes Instrument zur strategischen Kompetenzentwicklung zur Verfügung, welches sich insbesondere durch eine einfache Handhabbarkeit und transparente Anwendbarkeit auszeichnet. Die KMT wurde im Forschungsprojekt StraKosphere bislang in vier produzierenden KMU (darunter bei der in diesem Beitrag vorgestellten Firma alutec) unter wissenschaftlicher Anleitung implementiert und erprobt (für Erfahrungsberichte dazu siehe die Fallstudien von Dickhardt und Dickhardt 2018; Kaiser und Kaiser 2018; Kretz 2018; Wagner 2018). In einem Fall (Kaiser und Kaiser 2018) fand eine datenbankgestützte Umsetzung der KMT und Einbindung in das betriebseigene EDV-System statt. Trotz der bisherigen positiven Erfahrungen bei der Implementierung der KMT ist auch zu erwähnen, dass die Einführung von Instrumentarien zur strategischen Kompetenzentwicklung in KMU oftmals mit Skepsis betrachtet wird. Gründe dafür sind vor allem unklare Rentabilitätserwartungen sowie als prioritär angesehene, spontane Anforderungen des Alltagsgeschäfts (Virgillito 2018). Die KMT erfüllt neben praxisbezogenen Evaluationskriterien in ersten Ansätzen auch die psychometrischen Kriterien der Inhaltsvalidität sowie der Augenscheinvalidität - eine darüberhinausgehende Validierung, die in zukünftigen Studien erfolgen könnte, steht jedoch bislang aus.
Die KMT bietet gegenüber den zuvor präsentierten Kompetenzmessinstrumenten folgende Vorteile: Im Gegensatz zur Kompetenzmatrix von North et al. (2013) ist unter anderem eine Verrechnung einzelner Teilkompetenzen sowie ein Soll-Ist-Abgleich möglich. Gegenüber der prozessbezogenen Kompetenzmatrix von Hasenau et al. (2013) wird die Kompetenzeinstufung bei der KMT von den Nutzerinnen und Nutzern über standardisierte Einstufungsschlüssel vorgenommen, welche sowohl die Objektivität als auch die Usability des Instruments verbessert. Das Tool-PE-Instrumentarium von Galiläer und Wende (2008) unterstützt im Gegensatz zur KMT keine quantitative Bestimmung des Sollwertes und bietet darüber hinaus keine direkten Verknüpfungsmöglichkeiten für (außerberuflich) erworbene Kompetenzen und Qualifikationen an. Dadurch weist die KMT gegenüber den genannten Instrumenten insgesamt eine höhere Handhabbarkeit auf und lässt sich einfacher in ein datenbankgestütztes System überführen.

Damit die Implementierung eines Kompetenzentwicklungsinstrumentes wie der KMT gelingt, sind laut Virgillito (2018) folgende Zielgruppen im Unternehmen zu berücksichtigen: Die Geschäftsleitung sollte für Transparenz und Partizipationsmöglichkeiten sorgen, der Personalbereich sollte gestärkt und in den Strategieprozess sinnvoll eingebunden werden. Da der unteren Führungsebene eine wichtige Hebelwirkung und Multiplikatorfunktion zukommt, sollten die Führungskompetenzen der Schichtführerinnen und Schichtführer, Teamleiterinnen und Teamleiter etc. überprüft und gestärkt werden. Bei den an- und ungelernten Beschäftigten sollten Prüfungsängste, Weiterbildungsscheu, Sprach- und Verständnisschwierigkeiten ernst genommen und auch Lese- und Schreibschwierigkeiten berücksichtigt werden, um eine Überforderung der Beschäftigten zu vermeiden und Lernmotivation herzustellen. Nicht zuletzt sollte das oft unterschätzte Unterstützungspotential des Betriebsrates durch eine kooperative Einbindung nicht ungenutzt bleiben. Für die zu berücksichtigenden rechtlichen Rahmenbedingungen bei der Implementierung der KMT mit besonderer Berücksichtigung der betrieblichen Mitbestimmung sei auf das entsprechende Kapitel bei Decius et al. (2018) verwiesen.

Wichtig ist außerdem, auf der Ebene der Unternehmenskultur zu erreichen, dass die qualifikatorische Förderung und Kompetenzentwicklung der Beschäftigten als ein von allen betrieblichen Akteuren zu beachtendes strategisches Unternehmensziel angesehen wird. Dies geht einher mit einer positiven Lernkultur in der Organisation (Kortsch und Kauffeld 2019; Schaper et al. 2006). Zudem müssen die Ressourcen zur Kompetenzentwicklung bereitgestellt werden (Virgillito 2018) und das Vorgehen zur Kompetenzbedarfserfassung und -entwicklung organisatorisch in der betrieblichen Aufbau- und Ablauforganisation verankert sein (Decius et al. 2018). 
Unter Berücksichtigung dieser Rahmenbedingungen kann die KMT als ein Instrumentarium des Kompetenzmanagements KMU dabei unterstützen, die sich durch zunehmende Dynamisierungs- und Digitalisierungsprozesse wandelnden Anforderungen an die Beschäftigten frühzeitig zu erfassen. Auf dieser Basis können nicht nur zielführende Qualifizierungsmaßnahmen, sondern auch adäquate Anpassungen der Arbeitsorganisation und der Arbeits- und Aufgabengestaltung in Abstimmung mit den veränderten organisatorischen und technischen Veränderungen vorgenommen werden. Mit Hilfe der KMT können KMU konkret sowohl Sollprofile auf Grundlage von Anforderungsanalysen festlegen als auch die Istwerte der Beschäftigten systematisch identifizieren, dokumentieren und Qualifizierungsbedarfe feststellen. Um eine objektive und ökonomisch effiziente Auswertung zu gewährleisten, besteht die Möglichkeit einer Umsetzung der KMT als datenbankgestütztes Tool. Als Zusatzelemente ist eine Erfassung von sprachlichen Kompetenzen und außerberuflich erworbenen Kompetenzen möglich. Ein Implementierungsund Anwendungsleitfaden garantiert eine Anwendung auch ohne wissenschaftliche Anleitung.

Die KMT bietet insgesamt ein für die Bedarfe von KMU in Hinblick auf ein strategisches Kompetenzmanagement angepasstes, methodisch ausgearbeitetes, nützliches, aufwandsgerechtes und beherrschbares Instrumentarium an, das auch von Personalverantwortlichen und Führungskräften ohne einen spezifischen professionellen Hintergrund im Bereich der Personal- und Kompetenzentwicklung eingesetzt werden kann. Mit Hilfe der KMT können Personalverantwortliche die Kompetenzen der Beschäftigten erfassen und Abweichungen von (zukünftigen) Anforderungsprofilen frühzeitig erkennen. Die KMT kann auch zur systematischen Durchführung von Personalentwicklungsgesprächen herangezogen sowie für Einsatz-, Urlaubs- und Vertretungsplanungen und langfristige personelle Umbrüche verwendet werden.

Zusatzmaterial online Zusätzliche Informationen sind in der OnlineVersion dieses Artikels (https://doi.org/10.1007/s11612-021-00566w) enthalten.

Funding Open Access funding enabled and organized by Projekt DEAL.

Open Access Dieser Artikel wird unter der Creative Commons Namensnennung 4.0 International Lizenz veröffentlicht, welche die Nutzung, Vervielfältigung, Bearbeitung, Verbreitung und Wiedergabe in jeglichem Medium und Format erlaubt, sofern Sie den/die ursprünglichen Autor(en) und die Quelle ordnungsgemäß nennen, einen Link zur Creative Commons Lizenz beifügen und angeben, ob Änderungen vorgenommen wurden.

Die in diesem Artikel enthaltenen Bilder und sonstiges Drittmaterial unterliegen ebenfalls der genannten Creative Commons Lizenz, sofern sich aus der Abbildungslegende nichts anderes ergibt. Sofern das betreffende Material nicht unter der genannten Creative Commons Lizenz steht und die betreffende Handlung nicht nach gesetzlichen Vorschriften erlaubt ist, ist für die oben aufgeführten Weiterverwendungen des Materials die Einwilligung des jeweiligen Rechteinhabers einzuholen.

Weitere Details zur Lizenz entnehmen Sie bitte der Lizenzinformation auf http://creativecommons.org/licenses/by/4.0/deed.de.

\section{Literatur}

Abel, J., \& Wagner, P. S. (2017). Industrie 4.0: Mitarbeiterqualifizierung in KMU. wt-Werkstattstechnik online, 107, 134-140.

Abel, J., Decius, J., Güth, S., \& Schaper, N. (2016). Kompetenzentwicklung bei Un- und Angelernten in nicht-forschungsintensiven KMU - Status quo und Zukunft einer strategischen Notwendigkeit. Betriebspraxis \& Arbeitsforschung, 228, 41-50.

Bimrose, J., Mulvey, R., \& Brown, A. (2016). Low qualified and low skilled: the need for context sensitive careers support. British Journal of Guidance \& Counselling, 44(2), 145-157. https://doi. org/10.1080/03069885.2016.1145190.

Bishop, D. (2017). Context, agency and professional workplace learning. Education + Training, 59(5), 516-533. https://doi.org/10. 1108/et-07-2016-0129.

Buller, P.F., \& McEvoy, G. M. (2012). Strategy, human resource management and performance: sharpening line of sight. Human Resource Management Review, 22(1), 43-56. https://doi.org/10. 1016/j.hrmr.2011.11.002.

Campion, M. A., Fink, A. A., Ruggeberg, B. J., Carr, L., Phillips, G. M., \& Odman, R.B. (2011). Doing competencies well: best practices in competency modeling. Personnel Psychology, 64(1), 225-262. https://doi.org/10.1111/j.1744-6570.2010.01207.x.

Cascio, W.F., \& Montealegre, R. (2016). How technology is changing work and organizations. Annual Review of Organizational Psychology and Organizational Behavior, 3, 349-375. https://doi. org/10.1146/annurev-orgpsych-041015-062352.

Coetzer, A., Kock, H., \& Wallo, A. (2017). Distinctive characteristics of small businesses as sites for informal learning. Human Resource Development Review, 16(2), 111-134. https://doi.org/10.1177/ 1534484317704291.

Coetzer, A., Susomrith, P., \& Ampofo, E. T. (2020). Opportunities to participate in formal and informal vocational learning activities and work-related outcomes in small professional services businesses. Journal of Vocational Education \& Training, 72(1), 88-114. https://doi.org/10.1080/13636820.2019.1584637.

Decius, J. (2020). Informelles Lernen im Kontext industrieller Arbeit - Konzeptualisierung, Operationalisierung, Antezedenzien und Lernergebnisse. Manteltext der kumulativen Dissertationsschrift. Paderborn: Universität Paderborn. https://doi.org/10. 17619/UNIPB/1-1072.

Decius, J., \& Schaper, N. (2017). The Competence Management Tool (CMT) - A new instrument to manage competences in small and medium-sized manufacturing enterprises. Procedia Manufacturing, 9, 376-383. https://doi.org/10.1016/j.promfg.2017.04.041.

Decius, J., Schaper, N., \& Bender, E. (2018). Lösungsansatz und Gestaltungsoptionen eines Kompetenzmessinstruments für nichtforschungsintensive KMU. In D. Horvat, N. Schaper, A. Virgillito \& J. Decius (Hrsg.), Gestaltung eines strategischen Kompetenzmanagements - Lösungsansätze und Instrumente für nichtforschungsintensive, mittelständische Unternehmen (S. 59-77). Stuttgart: Fraunhofer IRB.

Decius, J., Schaper, N., \& Seifert, A. (2019). Informal workplace learning: development and validation of a measure. Human Resource Development Quarterly, 30(4), 495-535. https://doi.org/10.1002/ hrdq. 21368.

Dickhardt, R., \& Dickhardt, R. (2018). Fallstudie der SMK Röhrsdorf GmbH. In D. Horvat, N. Schaper, A. Virgillito \& J. Decius (Hrsg.), Gestaltung eines strategischen Kompetenzmanagements 
- Lösungsansätze und Instrumente für nichtforschungsintensive, mittelständische Unternehmen (S. 109-119). Stuttgart: Fraunhofer IRB.

Döring, N., \& Bortz, J. (2016). Forschungsmethoden und Evaluation in den Sozial- und Humanwissenschaften. Heidelberg: Springer. https://doi.org/10.1007/978-3-642-41089-5.

Erpenbeck, J., \& von Rosenstiel, L. (Hrsg.). (2003). Handbuch Kompetenzmessung. Stuttgart: Schäffer-Poeschel.

Fischer, M., Huber, K., Mann, E., \& Röben, P. (2015). Anerkennung informell erworbener Kompetenzen in der Metall- und Elektroindustrie in Baden-Württemberg. In E. Severing \& M. Baethge (Hrsg.), Sicherung des Fachkräftepotenzials durch Nachqualifzierung. Befunde - Konzepte - Forschungsbedarf (S. 93-113). Bielefeld: Bertelsmann.

Galiläer, L., \& Wende, R. (2008). Früherkennung im Betrieb - Instrumente und Methoden zur Erhebung des aktuellen und zukünftigen Qualifikationsbedarfs. Qualifikationstrends - Erkennen, Aufbereiten, Transferieren. Wirtschaft und Bildung, 47, 81-106.

Gustavsson, M. (2007). The potential for learning in industrial work. Journal of Workplace Learning, 19(7), 453-463. https://doi.org/ $10.1108 / 13665620710819393$.

Güth, S., Decius, J., Horvat, D., Schaper, N., \& Virgillito, A. (2018). Strategisches Kompetenzmanagement von Produktionsbeschäftigen - Innovations- und Wachstumsimpulse in nicht-forschungsintensiven kleinen und mittleren Unternehmen. In D. Ahrens \& G. Molzberger (Hrsg.), Kompetenzentwicklung in analogen und digitalisierten Arbeitswelten. Kompetenzmanagement in Organisationen (S. 31-49). Berlin: Springer. https://doi.org/10.1007/ 978-3-662-54956-8 4.

Hadjimanolis, A. (1999). Barriers to innovation for SMEs in a small less developed country (Cyprus). Technovation, 19(9), 561-570. https://doi.org/10.1016/s0166-4972(99)00034-6.

Hamborg, K.-C., \& Schaper, N. (2018). Analyse, Bewertung und Gestaltung von Aufgaben, Arbeitstätigkeiten und Arbeitssystemen. In S. Greif \& K.-C. Hamborg (Hrsg.), Methoden der Arbeits-, Organisations- und Wirtschaftspsychologie. Enzyklopädie der Psychologie (S. 1-79). Göttingen: Hogrefe.

Harteis, C. (2018). Machines, change and work: an educational view on the digitalization of work. In C. Harteis (Hrsg.), The impact of digitalization in the workplace (S. 1-10). Cham: Springer. https:// doi.org/10.1007/978-3-319-63257-5_1.

Hasenau, K., Sende, C., \& Nick, C. (2013). Erstellung prozessbezogener Kompetenzmatrizen in produzierenden KMU. In C. Schlick (Hrsg.), Handreichungen für die betriebliche Praxis (S. 1-8). Aachen: Institut für Arbeitswissenschaft (IAW) der RWTH Aachen.

Heil, L., Huber, J., Müller, S., Ness, H., \& Seitz, C. (2011). KMUKompetenzbuch. Entwicklung und Erprobung eines Instrumentes zur Kompetenzerfassung in kleinen und mittleren Unternehmen (Projektabschlussbericht). Frankfurt: Deutsches Institut für Internationale Pädagogische Forschung.

Hof, C. (2009). Lebenslanges Lernen: Eine Einführung (Grundriss der Pädagogik/Erziehungswissenschaft, Band 4). Stuttgart: Kohlhammer.

Horvat, D., Som, O., \& Moll, C. (2018). Leitfaden zur Umsetzung eines strategischen Kompetenzmanagements. In D. Horvat, N. Schaper, A. Virgillito \& J. Decius (Hrsg.), Gestaltung eines strategischen Kompetenzmanagements - Lösungsansätze und Instrumente für nichtforschungsintensive, mittelständische Unternehmen (S. 29-58). Stuttgart: Fraunhofer IRB.

Illeris, K. (2006). Lifelong learning and the low-skilled. International Journal of Lifelong Education, 25(1), 15-28. https://doi.org/10. 1080/02601370500309451.

Kaiser, W., \& Kaiser, S. (2018). Fallstudie der Friedr. Freek GmbH. In D. Horvat, N. Schaper, A. Virgillito \& J. Decius (Hrsg.), Gestaltung eines strategischen Kompetenzmanagements - Lösungsansätze und Instrumente für nichtforschungsintensive, mittelständische Unternehmen (S. 97-108). Stuttgart: Fraunhofer IRB.
Kauffeld, S. (2006). Kompetenzen messen, bewerten, entwickeln. Stuttgart: Schäffer-Poeschel.

Kauffeld, S., \& Frerichs, F. (2018). Kompetenzmanagement in kleinen und mittelständischen Unternehmen. Eine Frage der Betriebskultur? Berlin: Springer. https://doi.org/10.1007/978-3-662-548301

Kauffeld, S., \& Grote, S. (2019). Personalentwicklung. In S. Kauffeld (Hrsg.), Arbeits-, Organisations- und Personalpsychologie für Bachelor (S. 167-210). Berlin, Heidelberg, New York: Springer. https://doi.org/10.1007/978-3-662-56013-6_7.

Kauffeld, S., \& Paulsen, H. (2018). Kompetenzmanagement in Unternehmen. Kompetenzen beschreiben, messen, entwickeln und nutzen. Stuttgart: Kohlhammer.

Kaufhold, M. (2006). Kompetenz und Kompetenzerfassung. Analyse und Beurteilung von Verfahren der Kompetenzerfassung. Wiesbaden: Springer.

Kortsch, T., \& Kauffeld, S. (2019). Validation of a German version of the dimensions of the learning organization questionnaire (DLOQ) in German craft companies. Zeitschrift für Arbeits- und Organisationspsychologie, 63(1), 15-31. https://doi.org/10.1026/ 0932-4089/a000282.

Kortsch, T., Paulsen, H., \& Kauffeld, S. (2018). Unterstützungskultur trifft auf digitale Lösungen: Kompetenzentwicklung mit dem Kompetenz-Navi optimieren. In S. Kauffeld \& F. Frerichs (Hrsg.), Kompetenzmanagement in kleinen und mittelständischen Unternehmen: Eine Frage der Betriebskultur? (S. 181-193). Berlin: Springer. https://doi.org/10.1007/978-3-662-54830-1_11.

Kraiger, K., \& Ford, J. K. (2021). The science of workplace instruction: learning and development applied to work. Annual Review of $\mathrm{Or}$ ganizational Psychology and Organizational Behavior, 8, 45-72. https://doi.org/10.1146/annurev-orgpsych-012420-060109.

Kretz, K. (2018). Fallbeispiel alutec metal innovations GmbH \& Co. KG. In D. Horvat, N. Schaper, A. Virgillito \& J. Decius (Hrsg.), Gestaltung eines strategischen Kompetenzmanagements - Lösungsansätze und Instrumente für nichtforschungsintensive, mittelständische Unternehmen (S. 120-130). Stuttgart: Fraunhofer IRB.

Lundkvist, A.H., \& Gustavsson, M. (2018). Conditions for employee learning and innovation-interweaving competence development activities provided by a workplace development programme with everyday work activities in SMEs. Vocations and Learning, 11(1), 45-63. https://doi.org/10.1007/s12186-017-9179-6.

Mansfield, R. S. (1996). Building competency models: approaches for HR professionals. Human Resource Management, 35(1), 7-18. https://doi.org/10.1002/(SICI)1099-050X(199621)35:1\%3C7:: AID-HRM1\%3E3.0.CO;2-2.

Mesaros, L., Vanselow, A., \& Weinkopf, C. (2009). Fachkräftemangel in KMU - Ausmaß, Ursachen und Gegenstrategien. WISO Diskurs. Bonn: Friedrich-Ebert-Stiftung, Abteilung Wirtschafts- und Sozialpolitik.

Moll, C., \& Weidner, N. (2018). Strategisches Kompetenzmanagement in nicht-forschungsintensiven KMU. In D. Horvat, N. Schaper, A. Virgillito \& J. Decius (Hrsg.), Gestaltung eines strategischen Kompetenzmanagements - Lösungsansätze und Instrumente für nichtforschungsintensive, mittelständische Unternehmen (S. 9-28). Stuttgart: Fraunhofer IRB.

Moosbrugger, H., \& Kelava, A. (2012). Testtheorie und Fragebogenkonstruktion (2. Aufl.). Berlin, Heidelberg, New York: Springer. https://doi.org/10.1007/978-3-642-20072-4.

Noe, R. A., Clarke, A. D., \& Klein, H. J. (2014). Learning in the twenty-first-century workplace. Annual Review of Organizational Psychology and Organizational Behavior, 1(1), 245-275. https://doi. org/10.1146/annurev-orgpsych-031413-091321.

North, K., Reinhardt, K., \& Sieber-Suter, B. (2013). Kompetenzmanagement in der Praxis. Wiesbaden: Springer Gabler.

Paulsen, H., \& Kauffeld, S. (2019). Kompetenzmanagement in Organisationen: Ein Beitrag zur Laufbahnentwicklung. In D. Spurk \& 
S. Kauffeld (Hrsg.), Handbuch Karriere und Laufbahnmanagement (S. 511-542). Berlin: Springer. https://doi.org/10.1007/9783-662-45855-6_21-1.

Perkins, G. (2018). How does self-direction within learning operate to affect idea generation in small-medium enterprise contexts? $\mathrm{Hu}$ man Resource Development Quarterly, 28(1), 1-22. https://doi. org/10.1002/hrdq.21326.

Rammer, C., Berger, M., Doherr, T., Hud, M., Iferd, Y., Krieger, B., Peters, B., Schubert, T., \& Von der Burg, J. (2018). Indikatorenbericht zur Innovationserhebung 2017. http://ftp.zew.de/pub/zewdocs/mip/17/mip_2017.pdf. Zugegriffen: 28. Jan. 2021.

Regan, E., \& Delaney, C. (2011). Brave new workplace: The impact of technology on location and job structures. In M. Malloch, L. Cairns, K. Evans \& B. N. O'Connor (Hrsg.), The SAGE handbook of workplace learning (S. 431-442). London: SAGE. https:// doi.org/10.4135/9781446200940.n32.

Sanchez, J.I., \& Levine, E. L. (2009). What is (or should be) the difference between competency modeling and traditional job analysis? Human Resource Management Review, 19(2), 53-63. https://doi. org/10.1016/j.hrmr.2008.10.002.

Schaper, N. (2019a). Aus- und Weiterbildung: Konzepte der Trainingsforschung. In F. Nerdinger, G. Blickle \& N. Schaper (Hrsg.), Arbeits- und Organisationspsychologie (4. Aufl. S. 509-539). Berlin: Springer. https://doi.org/10.1007/978-3-662-56666-4_26.

Schaper, N. (2019b). Neue Formen der Arbeit: Das Beispiel Telekooperation. In F. Nerdinger, G. Blickle \& N. Schaper (Hrsg.), Arbeits- und Organisationspsychologie (4. Aufl. S. 601-628). Berlin: Springer. https://doi.org/10.1007/978-3-662-56666-4_29.

Schaper, N., Friebe, J., Wilmsmeier, A., \& Hochholdinger, S. (2006). Ein Instrument zur Erfassung unternehmensbezogener Lernkulturen - das Lernkulturinventar (LKI). In R. Rapp, P. Sedlmeier \& G. Zunker-Rapp (Hrsg.), Perspectives on cognition. A Festschrift for Manfred Wettler (S. 175-198). Lengerich: Pabst Science Publishers.

Shippmann, J. S., Ash, R. A., Batjtsta, M., Carr, L., Eyde, L.D., Hesketh, B., Sanchez, J.I., et al. (2000). The practice of competency modeling. Personnel Psychology, 53(3), 703-740. https://doi.org/ 10.1111/j.1744-6570.2000.tb00220.x.

Som, O., \& Horvat, D. (2018). Einleitung. In D. Horvat, N. Schaper, A. Virgillito \& J. Decius (Hrsg.), Gestaltung eines strategischen Kompetenzmanagements - Lösungsansätze und Instrumente für nichtforschungsintensive, mittelständische Unternehmen (S. 1-8). Stuttgart: Fraunhofer IRB.

Sonntag, K., Schaper, N., \& Benz, D. (1999). Leitfaden zur qualitativen Personalplanung bei technisch-organisatorischen Innovationen (LPI). In H. Dunckel (Hrsg.), Handbuch psychologischer Arbeitsanalyseverfahren (S. 285-317). Zürich: vdf.

Stevens, G.W. (2013). A critical review of the science and practice of competency modeling. Human Resource Development Review, 12(1), 86-107. https://doi.org/10.1177/2F1534484312456690.

Trim, J. L., North, B., Coste, D., Sheils, J., Quetz, J., Schneider, G., de l'Europe, C., et al. (2001). Gemeinsamer europäischer Referenzrahmen für Sprachen: lernen, lehren, beurteilen. Berlin: Langenscheidt.

Virgillito, A. (2018). Einführung eines strategischen Kompetenzmanagements in nicht-forschungsintensiven KMU. In D. Horvat, N. Schaper, A. Virgillito \& J. Decius (Hrsg.), Gestaltung eines strategischen Kompetenzmanagements - Lösungsansätze und Instrumente für nichtforschungsintensive, mittelständische Unternehmen (S. 79-94). Stuttgart: Fraunhofer IRB.

Wagner, R.M. (2018). Topstar-Bericht StraKosphere - Top-DownKompetenzentwicklung in der Fertigungspraxis. In D. Horvat, N. Schaper, A. Virgillito \& J. Decius (Hrsg.), Gestaltung eines strategischen Kompetenzmanagements - Lösungsansätze und Instrumente für nichtforschungsintensive, mittelständische Unternehmen (S. 131-140). Stuttgart: Fraunhofer IRB.

Weinert, F.E. (2001). Vergleichende Leistungsmessung in Schulen eine umstrittene Selbstverständlichkeit. In F.E. Weinert (Hrsg.), Leistungsmessungen in Schulen (3. Aufl. S. 17-31). Weinheim/ Basel: Beltz.

Wong, K. Y., \& Aspinwall, E. (2004). Characterizing knowledge management in the small business environment. Journal of Knowledge Management, 8(3), 44-61. https://doi.org/10.1108/ 13673270410541033.

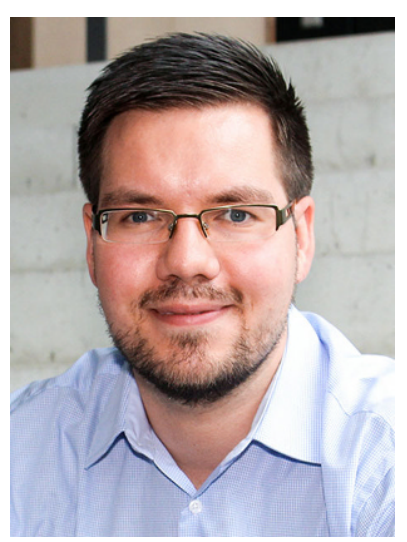

Dr. Julian Decius ist wissenschaftlicher Mitarbeiter am Lehrstuhl für Arbeits- und Organisationspsychologie der Universität Paderborn, wo er zum Thema des informellen Lernens im Kontext industrieller Arbeit promovierte. Er studierte Psychologie mit Vertiefungsrichtung Wirtschaftspsychologie an der Ruhr-Universität Bochum sowie der Lindenwood University, Missouri, USA. Sein Forschungsfokus liegt auf selbstreguliertem und informellem Lernen am Arbeitsplatz und im Studium, strategischer Kompetenzentwicklung in mittelständischen Unternehmen, Weiterbildungsformen unter Berücksichtigung von „Arbeit 4.0“ und „New Work“, sowie Kompetenzerfassung und -förderung bei Profi-Fußballspielern. Einblicke in die Praxis des angewandten Kompetenzmanagements erhielt er u.a. während des BMBFVerbundforschungsprojektes „StraKosphere“, an dem vier mittelständische Produktionsunternehmen beteiligt waren.

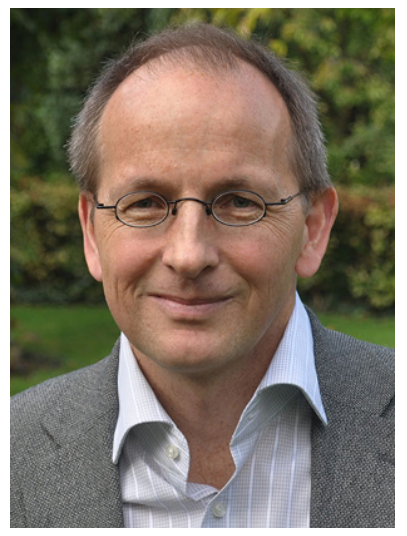

Prof. Dr. Niclas Schaper ist Professor für Arbeits- und Organisationspsychologie an der Universität Paderborn. Er hat verschiedene Forschungsprojekte zu Fragen des Kompetenzmanagements bei der strategischen Personalarbeit in KMUs und bei der Curriculumund Personalentwicklung im Hochschulkontext geleitet. Außerdem war er mit verschiedenen Teilprojekten an der Clusterförderung it's OWL zu Fragen von Industrie 4.0 und Arbeit 4.0 beteiligt. Aktuell beschäftigt er sich in einem BMBFProjekt im Kontext der Förderlinie „Zukunft der Arbeit“ mit Fragen der partizipativen und humangerechten Begleitung von KMUs bei der digitalen Transformation von Arbeitsprozessen. Seine Forschungsschwerpunkte und -interessen liegen in den Bereichen Kompetenzerfassung und -modellierung, betriebliche Laufbahngestaltung, betriebliches Weiterbildungsverhalten insbesondere in Form von informellen Lernaktivitäten im Arbeitskontext und seine Einflussfaktoren, Erfassung organisationaler Lernkulturen, Weiterentwicklung von Arbeits- und Anforderungsanalysen, Mitarbeiterbefragungen und organisationale Bindung von Mitarbeitern. 